\title{
1DV bottom boundary layer modeling under combined wave and current: Turbulent separation and phase lag effects
}

\author{
Katell Guizien, ${ }^{1,4}$ Marjolein Dohmen-Janssen, ${ }^{2}$ and Giovanna Vittori ${ }^{3}$ \\ Received 9 January 2002; revised 3 September 2002; accepted 21 November 2002; published 28 January 2003.
}

[1] On the basis of the Wilcox [1992] transitional $k$ - $\omega$ turbulence model, we propose a new $k$ - $\omega$ turbulence model for one-dimension vertical (1DV) oscillating bottom boundary layer in which a separation condition under a strong, adverse pressure gradient has been introduced and the diffusion and transition constants have been modified. This new turbulence model agrees better than the Wilcox original model with both a direct numerical simulation (DNS) of a pure oscillatory flow over a smooth bottom in the intermittently turbulent regime and with experimental data from Jensen et al. [1989], who attained the fully turbulent regime for pure oscillatory flows. The new turbulence model is also found to agree better than the original one with experimental data of an oscillatory flow with current over a rough bottom by Dohmen-Janssen [1999]. In particular, the proposed model reproduces the secondary humps in the Reynolds stresses during the decelerating part of the wave cycle and the vertical phase lagging of the Reynolds stresses, two shortcomings of all previous modeling attempts. In addition, the model predicts suspension ejection events in the decelerating part of the wave cycle when it is coupled with a sediment concentration equation. Concentration measurements in the sheet flow layer give indication that these suspension ejection events do occur in practice. INDEX TERMS: 4211 Oceanography: General: Benthic boundary layers; 4560 Oceanography: Physical: Surface waves and tides (1255); 4568 Oceanography: Physical: Turbulence, diffusion, and mixing processes; 4842 Oceanography: Biological and Chemical: Modeling; 4558 Oceanography: Physical: Sediment transport; KEYWORDS: turbulence, modeling, wave boundary layer, sediment dynamics

Citation: Guizien, K., M. Dohmen-Janssen, and G. Vittori, 1DV bottom boundary layer modeling under combined wave and current: Turbulent separation and phase lag effects, J. Geophys. Res., 108(C1), 3016, doi:10.1029/2001JC001292, 2003.

\section{Introduction}

[2] In coastal zones, the suspension associated to waves and currents in the bottom boundary layer can have an impact on both human activities and ecological equilibrium. Indeed, it is well known that suspension plays a major role in sediment transport and affects human works and biological species through morphodynamical changes, which may affect the stability of the former and destroy the habitats of the latter. Moreover suspension can also affect directly the life cycle of some species and hence play a role in their population dynamics. This is the case for instance for benthic invertebrates with planktonic larvae. Indeed, the larvae settlement on the bed may be limited by strong suspension events and lead to dramatic cut in the population. Studying the suspension dynamics under waves and currents is hence

\footnotetext{
${ }^{1}$ Laboratoire des Ecoulements Géophysiques et Industriels, Grenoble Cedex 9, France.

${ }^{2}$ Department of Civil Engineering, University of Twente, Enschede, Netherlands. Italy.

${ }^{3}$ Dipartimento di Ingegneria Ambientale, Università di Genova, Genoa,

${ }^{4}$ Now at Laboratoire d'Océanographie Biologique de Banyuls, Banyuls sur Mer Cedex, France.

Copyright 2003 by the American Geophysical Union. 0148-0227/03/2001JC001292\$09.00
}

of great interest, especially over flat bed since this corresponds to the more severe hydrodynamical conditions.

[3] As a conclusion of the MAST II G8-M Coastal Morphodynamics European project, some shortcomings in modeling sand transport by combined waves and currents have been identified which are reported by Davies et al. [1997]. In their paper, an intercomparison of experimental data with four research sediment transport models under combined waves and currents was presented. The four models mainly differed in the complexity of the turbulence closure schemes (from zero to two-equations) used to compute the eddy-viscosity in the bottom turbulent boundary layer. In Fredsøe's [1984] model, a time-dependent eddy viscosity is derived from integration of the momentum equation over the wave boundary layer, assuming a logarithmic velocity profile (zero-equation model). Ribberink and Al Salem [1995] used a time- and height-dependent eddy viscosity by extending Prandtl's mixing length theory to an unsteady flow (zero-equation model). $\mathrm{Li}$ and Davies [1996] used a $k$-equation model with a similarity $l$-scaling (one-equation model) and Huynh Than et al. [1994] used a $k-L$ model (two-equation model) to compute a time-varying eddy viscosity. The concentration is computed from a convection-diffusion equation in which vertical sediment diffusivity is assumed to be equal to the time-dependent eddy viscosity, except in the Huynh Than et al. model where 


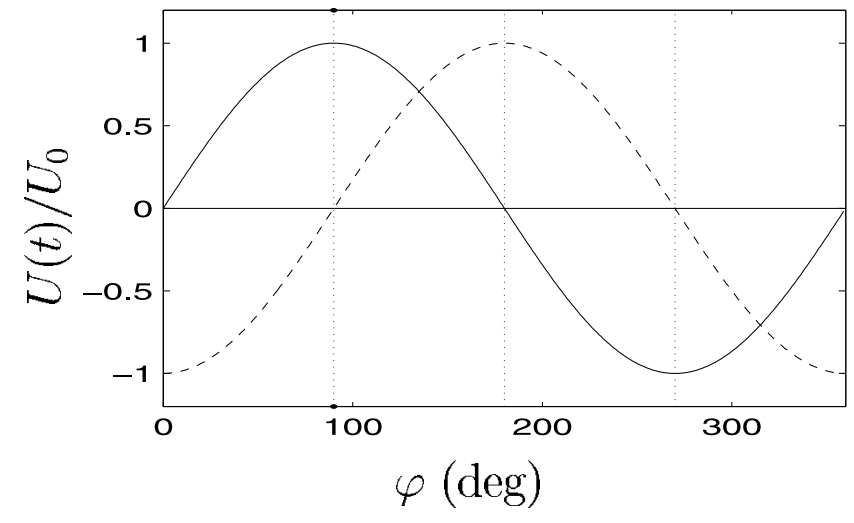

Figure 1. Phase $\varphi$ definition along the oscillatory part of the outer flow velocity $(-)$ and corresponding pressure gradient (- -).

turbulence damping is taken into account so that eddy viscosity and sediment diffusivity are related through some coupling terms. Despite the difference in the complexity of the turbulence closure, all the models show similar shortcomings when predictions are compared to flat bed experiments which correspond to strong wave plus current conditions ("sheet flow" regime).

[4] All the models lead to underestimation of the phase lag between concentration and velocity in the upper part of an oscillatory boundary layer and to unreliable estimates of sediment load predictions. Recent experiments in clear water (without sediment) by Dohmen-Janssen [1999] show a relevant phase lag over depth in Reynolds stress time series thus showing that the phase lag between concentration and velocity is partially inherent to the oscillatory boundary layer dynamics and not totally due to the sediment feedback on the turbulence structure. Therefore, efforts should be done to improve turbulence modeling for oscillatory boundary layers before working on flow and sediment coupling.

[5] In particular, none of the aforementioned models reproduce correctly the phase lag between Reynolds stress and velocity. This phase lag is related to the Reynolds stress vertical decay in the region far from the wall: the quicker it decays, the larger the phase lag is. In a recent paper, Sana and Tanaka [2000] present a comparison between five lowReynolds-number $k-\epsilon$ models and the direct numerical simulation (DNS) by Spalart and Baldwin [1989] for sinusoidal oscillatory flows over smooth beds. They show that the Jones and Launder's [1972] model provides better predictions of transition initiation and of the Reynolds stress vertical decay in the region far from the wall. These results suggest that the introduction of low-Reynolds-number modifications could improve the modeling of phase lag between Reynolds stress and velocity. However, it should also be pointed out that Jones and Launder's model underestimates the peak value of the turbulent kinetic energy and overestimates the bottom shear stress enhancement after transition. It can be concluded that none of the low-Reynoldsnumber modifications proposed in these five $k-\varepsilon$ models enable to predict correctly the whole dynamics of the oscillating boundary layer.

[6] A second shortcoming of the models considered by Davies et al. [1997] concerns concentration secondary peaks which are sometimes observed near flow reversal in experimental measurements close to the bottom [Katapodi et al., 1994; Dohmen-Janssen, 1999] and are not reproduced by models. Although the very sharp concentration peaks that show in the measurements close to the bottom may be partly caused by the measuring technique, there are indications that suspension ejection events really occur before flow reversal (see section 4). These may be attributed to shear instabilities in the wave boundary layer [Foster et al., 1994].

[7] The contribution of these secondary concentration peaks to the near-bed sediment load is limited, since they occur at a time when the velocity is nearly zero. However, the huge amount of sediment picked up from the bed around flow reversal, especially for fine sand, may affect turbulence and at the same time may be redistributed along time in the upper suspension layer. Hence, these concentration secondary peaks can be of great importance with respect to total sediment load predictions. Besides, such suspension ejection events can play a crucial role in benthic life.

[8] Savioli and Justesen [1996] proposed a modified condition for the reference concentration that enables to reproduce secondary peaks on the concentration time series with a standard (without low-Reynolds-number effects) $k-\epsilon$ model [Rodi, 1980] in a one-dimensional vertical (1DV) fully rough turbulent oscillating boundary layer model,
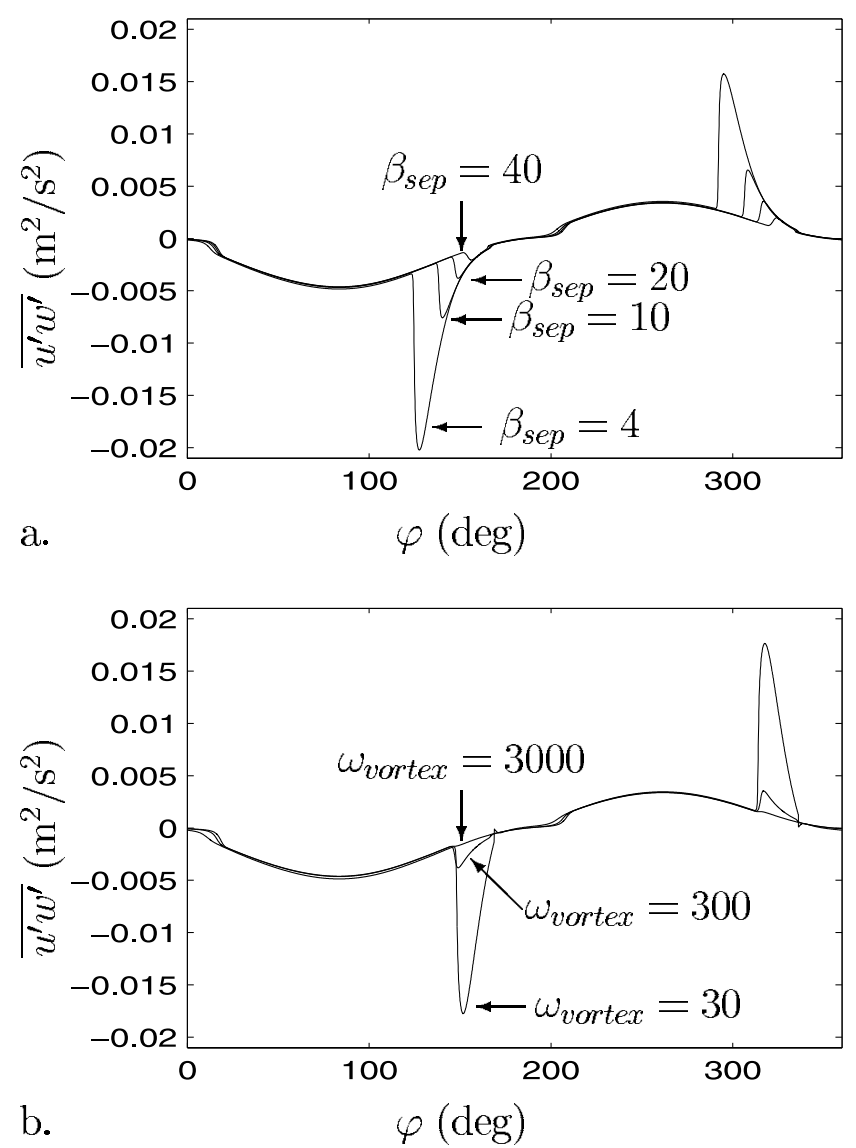

Figure 2. Phase-averaged Reynolds stress time series obtained using the new $k$ - $\omega$ model for (a) $\beta_{\text {sep }}$ ranging from 4 to $40\left(\omega_{\text {vortex }}=300\right)$ and (b) $\omega_{\text {vortex }}$ ranging from 30 to $3000\left(\beta_{\mathrm{sep}}=20\right)\left(R_{\delta}=2179, R_{e}=2.4 \times 10^{6}, A / k_{N}=3173\right)$. 


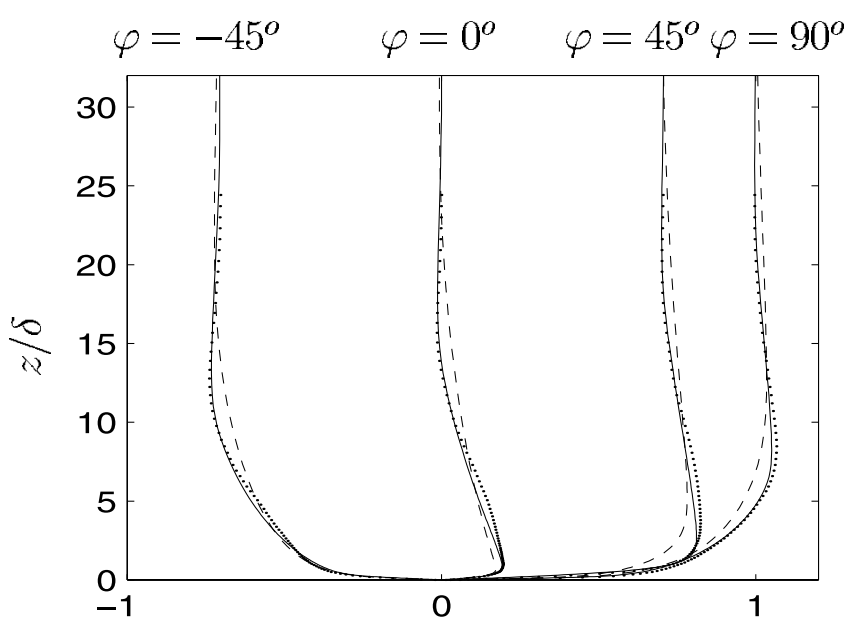

a.

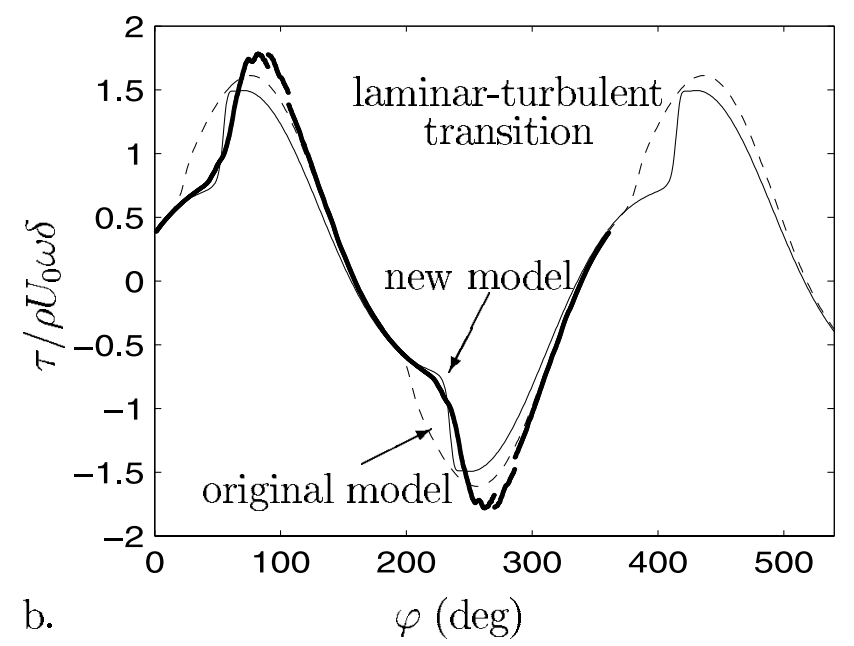

Figure 3. Velocity vertical profiles at different phases (a) and bottom shear stress time evolution (b) computed by DNS (...), the original Wilcox transitional $k-\omega$ model (- -) and the new $k$-w model $(-)$ for a sinusoidal outer flow $U=$ $U_{0} \sin (2 \pi t / T)$ with $T=4 \mathrm{~s}, U_{0}=1.1 \mathrm{~m} / \mathrm{s}$ over a smooth bottom $\left(R_{\delta}=1241, R_{e}=7.7 \times 10^{5}\right)$.

taking advantage of a narrow diffusivity peak just before flow reversal. A much smaller narrow peak, is also present near flow reversal in the eddy viscosity time series computed using a standard $k-\omega$ turbulence model [Wilcox, 1988], whereas a $k$-L turbulence model [Huynh Than et al., 1994] does not produce such peaks. However, although showing discrepancies on the eddy viscosity time series, the three turbulence models produce similar time series of the bottom shear stress, without any significant increase near flow reversal [Guizien et al., 2001]. In fact, differences in the eddy viscosity time series are due to the closures of the models, namely to the singularity in the behavior of the eddy viscosity, that reads $\nu_{T}=k / \omega$ in the $k-\omega$ model and $\nu_{T}=0.09 k^{2} / \epsilon$ in the $k$ - $\epsilon$ model. The singularity arises when $k$ and the other value $\omega$ or $\epsilon$ approach zero, for instance when the outer flow velocity decreases to zero during a wave cycle. At that phase, the instantaneous local Reynolds number decreases rapidly and the eddy viscosity strongly increases if the fully turbulent value for the model constants is applied. In steady boundary layers, it is well known that the constants used in $k-\epsilon$ standard models should be modified using low-Reynolds-number damping function to avoid the singular behavior of the eddy viscosity near the wall when computing the viscous sublayer. It is worth noticing that, in standard $k-\omega$ models, the viscous sublayer can be easily included for both smooth and rough bottom [Saffman, 1970], avoiding this latter near-wall singularity. In addition, under stationary conditions with an adverse pressure gradient and for low-Reynolds-numbers, standard $k-\omega$ models perform better than standard $k-\epsilon$ models [Wilcox, 1998]. This is consistent with the fact that the near-reversal eddy viscosity peak is smaller in the standard $k$ - $\omega$ computations than in the standard $k-\epsilon$ computations and that a much smaller time step (50 times smaller, strongly depending on the velocity amplitude) is required to deal with the singularity in computations with a standard $k-\epsilon$ model compared to computations performed with a standard $k-\omega$ model. However, introducing low-Reynolds-number effect in a $k-\epsilon$ turbulence model (e.g., Chien [1982] model, used by Thais et al. [1999]), the peak in the eddy viscosity time series for an oscillating boundary layer vanishes (L. Thais, personal communication, 1999). Similarly, when using the Wilcox [1992] transitional $k-\omega$ turbulence model, that includes low-Reynolds-number effect, the eddy viscosity time series for oscillating boundary layers do not present any peak.

[9] Recently, clear water experiments by Dohmen-Janssen [1999] shed a new light on this question. During these experiments, stronger turbulent activity was detected in the Reynolds stress time series close to the wall in the decelerating part of the wave cycle. This turbulence enhancement occurs at phases when the concentration secondary peaks are observed for the same hydrodynamical conditions. It should be outlined that fluctuations similar to the ones measured by Dohmen-Janssen were observed by Sleath [1987]. He also measured a $180^{\circ}$ phase shift of the phase of the Reynolds stress maximum at a certain height from the bed and explained it by assuming the existence of jets of fluids associated with vortex formation over the bottom roughness. He suggested that these jets of fluid would dominate the flow close to the wall whereas turbulence would dominate far from it. This explanation clearly implies that a detailed modeling of rough oscillating boundary layers should be three-dimensional and include a mechanism for vortex generation by bottom roughness. Even though such a sophisticated model is beyond the scope of this paper, it is clear that the strong turbulence activity which takes place during the decelerating phases of the cycle should be taken into account since it contributes to put more sediment in suspension. In this paper, starting from the Wilcox [1992] transitional $k$ - $\omega$ model, a new transitional $k-\omega$ turbulence model is proposed in order to improve the $1 \mathrm{DV}$ modeling of oscillating bottom boundary layers. A $k-\omega$ turbulence model is preferred to a $k-\epsilon$ one for its simplicity, its ability to include the viscous sublayer and for its good predictions under adverse pressure gradients, which occur during the decelerating phases of the wave cycle. The improvement brought to the Wilcox transitional $k-\omega$ model concerns vertical phase lagging and suspension ejection events. The damping of turbulence by the stratification is 

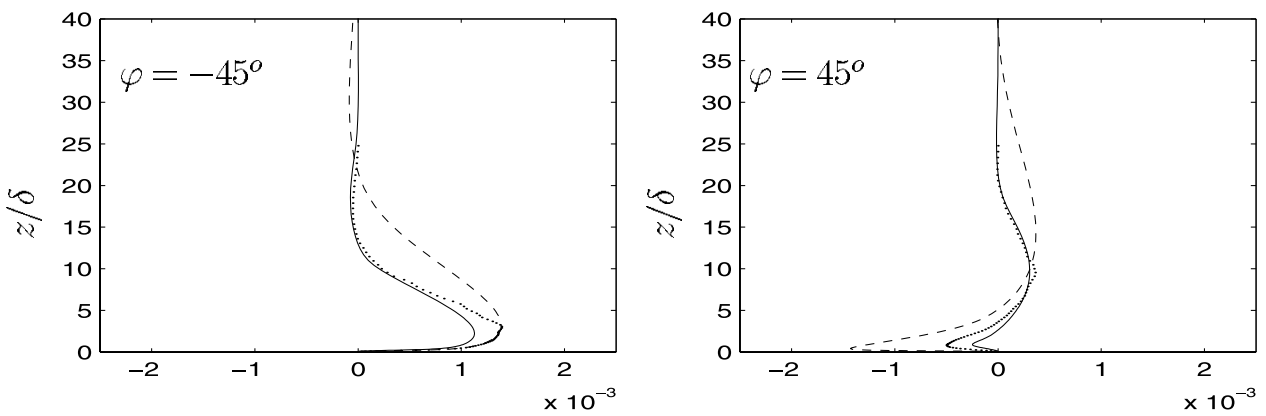

a.

$$
\overline{u^{\prime} w^{\prime}} / U_{0}^{2}
$$

c.

$$
\overline{u^{\prime} w^{\prime}} / U_{0}^{2}
$$
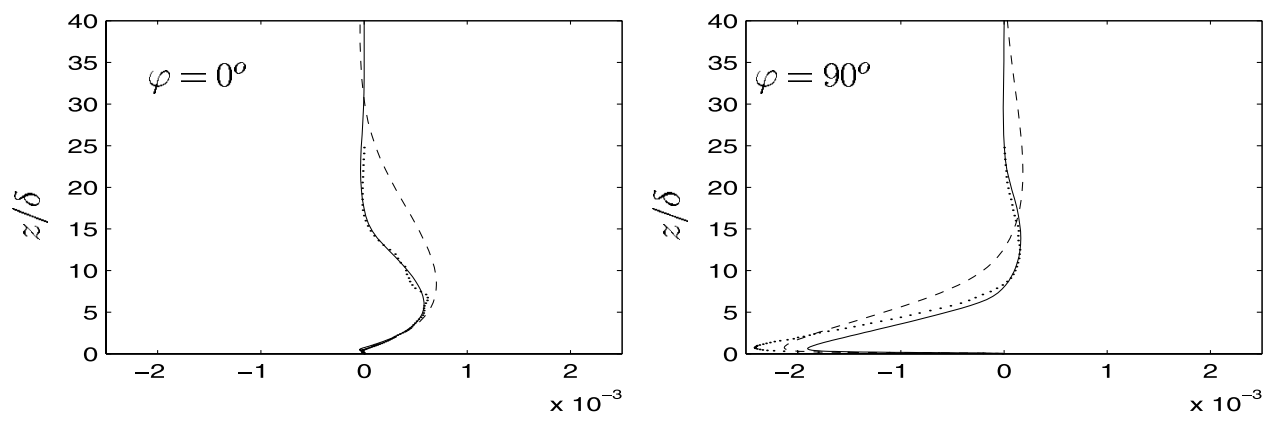

b.

$$
\overline{u^{\prime} w^{\prime}} / U_{0}^{2}
$$

d.

$\overline{u^{\prime} w^{\prime}} / U_{0}^{2}$

Figure 4. Reynolds stress vertical profiles at different phases: (a) $\varphi=-45^{\circ}$, (b) $\varphi=0^{\circ}$, (c) $\varphi=45^{\circ}$, and (d) $\varphi=90^{\circ}$ computed by DNS (...), the original Wilcox transitional $k$ - $\omega$ model (- -$)$ and the new $k-\omega$ model (-) for a sinusoidal outer flow $U=U_{0} \sin (2 \pi t / T)$ with $T=4 \mathrm{~s}, U_{0}=1.1 \mathrm{~m} / \mathrm{s}$ over a smooth bottom $\left(R_{\delta}=1241, R_{e}=7.7 \times 10^{5}\right)$.

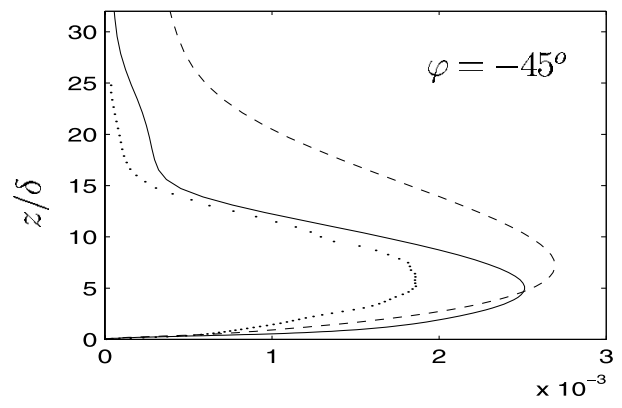

a.

$k / U_{0}^{2}$

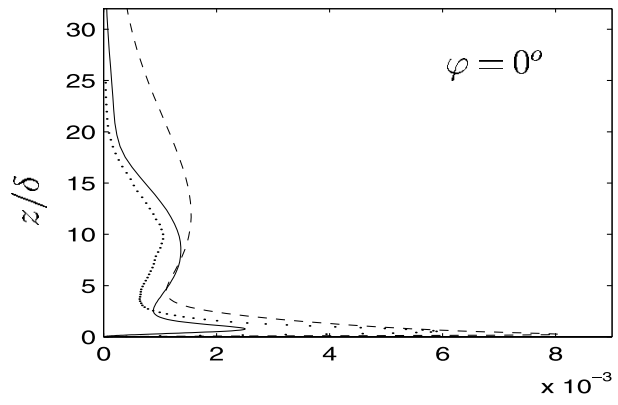

b.

$k / U_{0}^{2}$

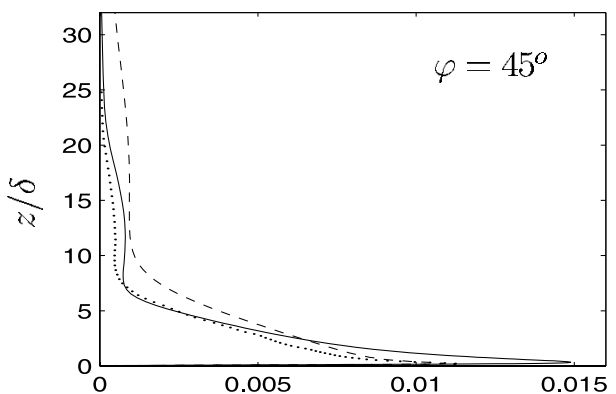

c.

$k / U_{0}^{2}$

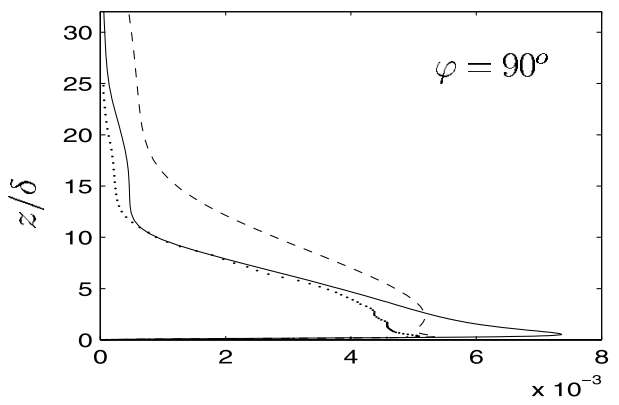

d.

$$
k / U_{0}^{2}
$$

Figure 5. Turbulent kinetic energy vertical profiles at different phases: (a) $\varphi=-45^{\circ}$, (b) $\varphi=0^{\circ}$, (c) $\varphi=$ $45^{\circ}$, and (d) $\varphi=90^{\circ}$ computed by DNS (...), the original Wilcox transitional $k$ - $\omega$ model (- - ), and the new $k$ - $\omega$ model (-) for a sinusoidal outer flow $U=U_{0} \sin (2 \pi t / T)$ with $T=4 \mathrm{~s}, U_{0}=1.1 \mathrm{~cm} / \mathrm{s}$ over a smooth bottom $\left(R_{\delta}=1241, R_{e}=7.7 \times 10^{5}\right)$. 


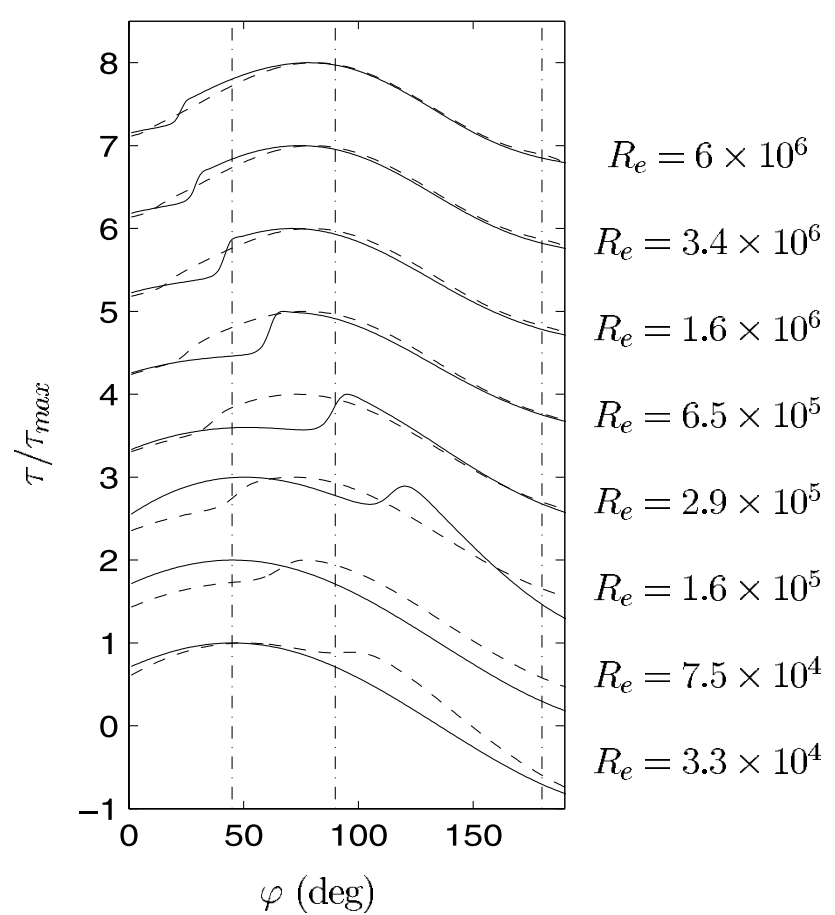

Figure 6. Half-period bottom shear stress time series showing laminar-turbulent transition for increasing Reynolds number predicted by the original Wilcox transitional $k$ - $\omega$ model (- $)$ and the new $k$ - $\omega$ model (-) for a sinusoidal outer flow over a smooth bottom.

also introduced. The model is presented in section 2. The ability of the new model to predict laminar-turbulent transition is tested for a pure oscillatory flow over a smooth bottom by comparison with direct numerical simulations in section 3.1 and with the experimental data from Jensen et al. [1989] in section 3.2. The model is then compared with experimental data in the rough turbulent regime for an oscillatory flow plus current in section 4.1 [Dohmen-Janssen, 1999]. Finally, concentration predictions corresponding to these latter hydrodynamical conditions are described in section 4.2.

\section{The New $k-\omega$ Model}

\subsection{Basic Formulation}

[10] The basis of the Reynolds Averaged Navier-Stokes (R.A.N.S.) model we use to compute the turbulent bottom boundary layer under an oscillatory flow (with or without current) is the transitional $k-\omega$ model devised by Wilcox [1992] in its 1DV formulation. In addition, turbulence damping by stratification is introduced into the original Wilcox formulation through coupling terms between turbulence and the density field $\rho(z, t)=\rho_{0}+C(z, t)\left(\rho_{s}-\rho_{0}\right)$ resulting from the sediment suspension $\left(\rho_{0}\right.$ is the fluid density, $\rho_{s}$ is the sediment density and $C(z, t)$ is the sediment concentration per volume). The coupling terms are similar to those introduced by Lewellen [1977] in a $k-L$ model. The hydrodynamical model (i.e., without sediment) is easily retrieved taking $\partial \rho / \partial z=0$.

[11] The horizontal velocity $u$ inside the boundary layer, the turbulent kinetic energy $k$ and the energy dissipation rate $\omega$ fulfill the following set of equations (1)-(6), where $U$ is the horizontal velocity outside the boundary layer (outer flow) and $\frac{\partial \bar{P}}{\partial x}$ is the mean pressure gradient generating the current (note that for pure oscillatory flow, $\frac{\partial P}{\partial x}=0$ ). In this 1DV formulation, we assume no $x$-dependence for all averaged quantities (no horizontal convective or diffusive transport) and no vertical velocity at the top of the boundary layer. These assumptions correspond strictly to the tunnel experiment conditions we will compare with in the next sections.

$$
\begin{gathered}
\frac{\partial u}{\partial t}=-\frac{1}{\rho_{0}} \frac{\partial \bar{P}}{\partial x}+\frac{\partial U}{\partial t}+\frac{\partial}{\partial z}\left(\left(\nu+\nu_{t}\right) \frac{\partial u}{\partial z}\right) \\
\frac{\partial k}{\partial t}=\nu_{t}\left(\frac{\partial u}{\partial z}\right)^{2}-\beta^{*} k \omega+\frac{\partial}{\partial z}\left[\left(\nu+\sigma^{*} \nu_{t}\right) \frac{\partial k}{\partial z}\right]+\frac{g}{\rho_{0}} \gamma_{t} \frac{\partial \rho}{\partial z} \\
\frac{\partial \omega}{\partial t}=\underbrace{\alpha \nu_{t} \frac{\omega}{k}\left(\frac{\partial u}{\partial z}\right)^{2}}_{\text {production }}-\underbrace{\beta \omega^{2}}_{\text {dissipation }}+\underbrace{\frac{\partial}{\partial z}\left(\left(\nu+\sigma \nu_{t}\right) \frac{\partial \omega}{\partial z}\right)}_{\text {diffusion }}+\underbrace{c_{0} \frac{\omega}{2 k} \frac{g}{\rho_{0}} \gamma_{t} \frac{\partial \rho}{\partial z}}_{\text {buoyancy }} \\
\nu_{t}=\alpha^{*} \frac{k}{\omega} \frac{1-C_{3} \Omega}{\left(1-C_{1} \Omega\right)\left(1-C_{2} \Omega\right)} \\
\gamma_{t}=\nu_{t} \frac{1-C_{2} \Omega}{\left(1-C_{3} \Omega\right)} \\
\Omega=2 \frac{g}{\rho_{0}} \frac{d \rho}{d z} \frac{4}{\omega^{2}}
\end{gathered}
$$

with

$$
\begin{aligned}
\alpha^{*}=\frac{\alpha_{0}^{*}+R e_{T} / R_{K}}{1+R e_{T} / R_{K}} ; \alpha & =\frac{13}{25} \frac{\alpha_{0}+\operatorname{Re}_{T} / R_{\omega}}{1+\operatorname{Re}_{T} / R_{\omega}}\left(\alpha^{*}\right)^{-1} ; \\
\beta^{*} & =\frac{9}{100} \frac{4 / 15+\left(R e_{T} / R_{\beta}\right)^{4}}{1+\left(\operatorname{Re}_{T} / R_{\beta}\right)^{4}}
\end{aligned}
$$

where $\operatorname{Re}_{T}=\frac{k}{\nu \omega}, \beta=\beta_{0}=\frac{9}{125}, \alpha_{0}^{*}=\frac{\beta_{0}}{3}, \alpha_{0}=\frac{1}{9}, R_{\omega}=2.95$, and $c_{0}=0.8$. It should be recalled here that, unlike most of the above coefficients, no simple argument can be found to estimate the values for $\sigma, \sigma^{*}, R_{K}$ and $R_{\beta}$. For given values for $R_{K}$ and $R_{\beta}$, there is a unique value of $R_{\omega}$ that yields the value measured by Nikuradse of the constant appearing in the law of the wall for smooth wall $C_{w}=5.0$. Hence, Wilcox [1992] proposed values for $R_{K}=6, R_{\beta}=8$ and $\sigma=\sigma^{*}=0.5$ that give the best agreement both with experiments and direct numerical simulations of steady boundary layers with and without adverse or favorable pressure gradient. However, he already outlined that taking a smaller value for $\sigma^{*}$ should improve the model's prediction for boundary layers with variable pressure gradient. Hence, on the basis of a preliminary analysis of the performances of the model we suggest to use the following values for oscillatory boundary layers (oscillatory pressure gradient): $\sigma=0.8, \sigma^{*}=$ $0.375, R_{K}=20$ and $R_{\beta}=27$. The original value for $R_{\omega}=$ 2.95 is kept and gives a constant for the law of the wall $C_{w}=7.6$ for a steady boundary layer in the smooth regime. These values provide better predictions than the values 


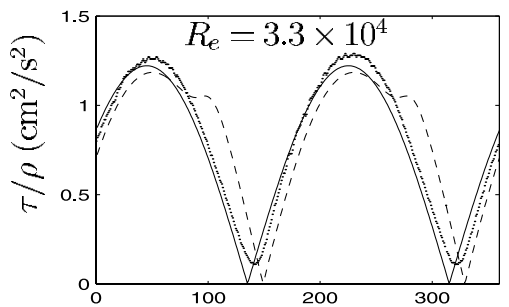

a.

$\varphi(\mathrm{deg})$

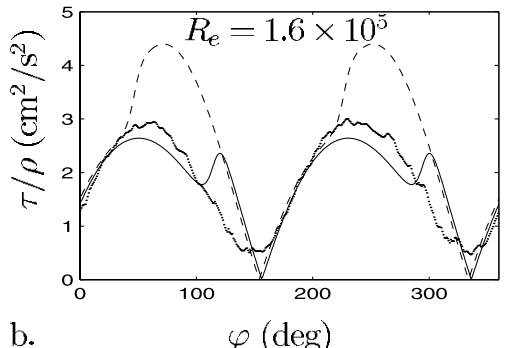

b.

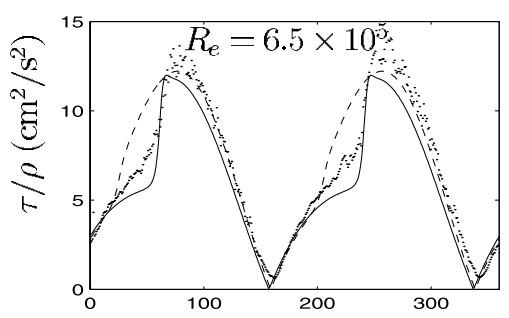

c.

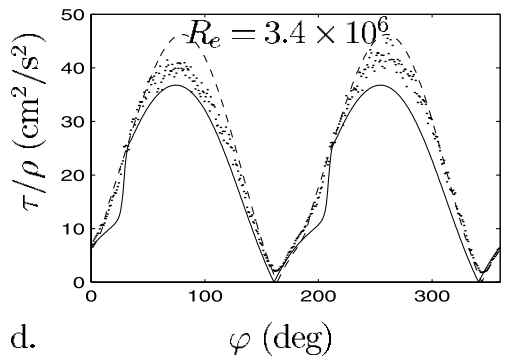

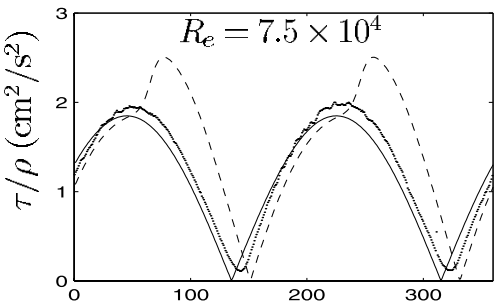

e. $\varphi(\mathrm{deg})$

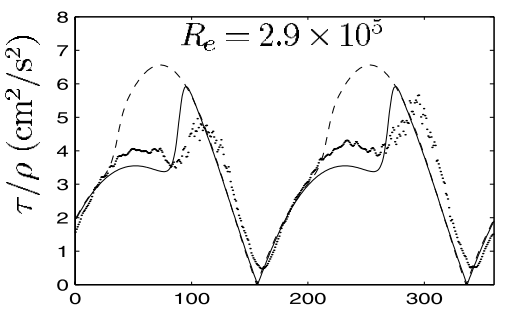

f.

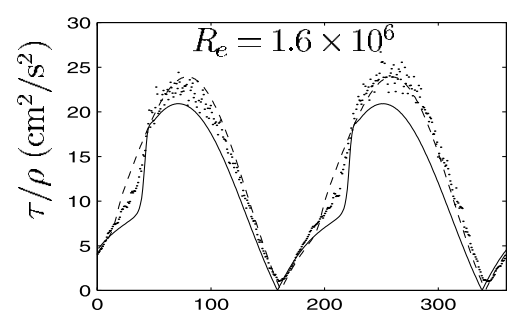

g. $\varphi(\mathrm{deg})$

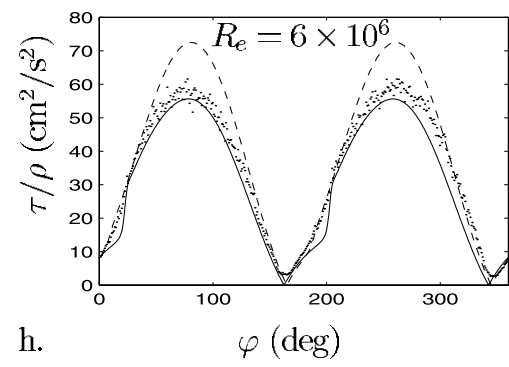

Figure 7. Bottom shear stress measured by Jensen et al. [1989] (...), the original Wilcox transitional $k$ $\omega$ model (- -) and the new $k$ - $\omega$ model (-) for a sinusoidal outer flow $U=U_{0} \sin (2 \pi t / T)$ with $T=9.72 \mathrm{~s}$ over a smooth bottom for different Reynolds number.

suggested by Wilcox when the results of the model are compared with a DNS computation of a pure oscillatory boundary layer in the smooth regime (see section 3.1). The equations (1)-(3) for $u, k$, and $\omega$ are solved using the implicit finite control volume method of Patankar [1980] on an exponential mesh with the following boundary conditions. At the bottom, we prescribe the true value of $k$ and $u$ [Saffman, 1970], meanwhile the value of $\omega$ is fixed depending on whether a smooth or rough wall should be modeled [Wilcox, 1998, p. 177]. At the top of the boundary layer we force the outer flow and the vanishing of turbulence. These conditions write:

1. At $z=0: u=0, k=0$ and $\omega_{\text {wall }}=\frac{u_{f}^{2}}{v} S_{R}$ with $S_{R}=(50 /$ $\left.k_{N}^{+}\right)^{2}$ if $k_{N}^{+}<25$ and $S_{R}=100 / k_{N}^{+}$if $k_{N}^{+} \geq 25$. The quantity $k_{N}^{+}$ is equal to $k_{N} u_{f} / v$ where $k_{N}$ is the Nikuradse roughness, $u_{f}=\sqrt{\tau / \rho_{0}}$ is the friction velocity and $\tau=\rho_{0}\left(\nu+\nu_{T}\right) \frac{\partial u}{\partial z}$ is the total bottom shear stress. Note that this boundary condition for a rough surface describes also a smooth surface provided $k_{N}^{+}$is small enough (in practice, $k_{N}^{+}<5$ ).

2. At $z=z_{h}: u=U=U_{0} \sin (2 \pi t / T)+U_{c}$ (where $U_{0}, T$ are the amplitude and period of the velocity oscillation and $U_{c}$ the current velocity) and $\partial k / \partial z=\partial \omega / \partial z=0$. For pure oscillatory flows $z=z_{h}$ is just outside the bottom boundary layer. When a steady current in a free surface flow is present, $z=z_{h}$ is the location of the free surface. Due to symmetries in tunnel experiments, $z=z_{h}$ is the location of the tunnel axis.

[12] When sediment suspension is considered, the following sediment concentration balance is solved together with the set of equations (1)-(6):

$$
\frac{\partial C}{\partial t}=\frac{\partial\left(w_{s} C\right)}{\partial z}+\frac{\partial}{\partial z}\left(\gamma_{t} \frac{\partial C}{\partial z}\right)
$$




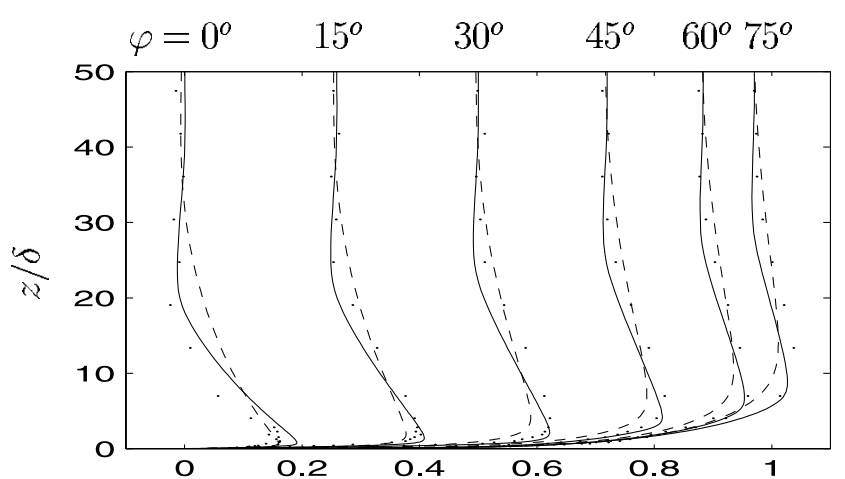

a. $u / U_{0}$
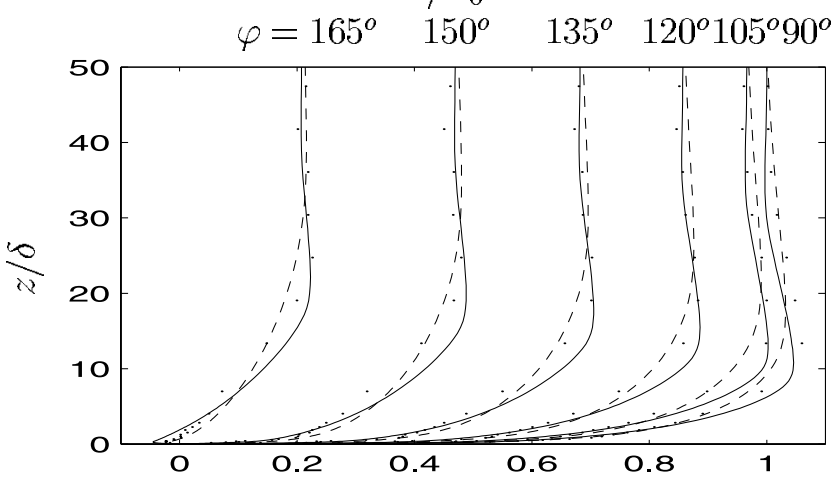

b.

$u / U_{0}$

Figure 8. Velocity vertical profiles at different phases during the accelerating part of the cycle (a) and during the decelerating part of the cycle (b) measured by Jensen et al. [1989, test 8] (..), computed with the original Wilcox transitional $k$ - $\omega$ model (- -) and the new $k$ - $\omega$ model (-) for a sinusoidal outer flow $U=U_{0} \sin (2 \pi t / T)$ with $T=9.72 \mathrm{~s}, U_{0}=$ $1.02 \mathrm{~m} / \mathrm{s}$ over a smooth bottom $\left(R_{\delta}=1789, R_{e}=1.6 \times 10^{6}\right)$.

where $w_{S}$ is the sediment settling velocity and the following boundary conditions are applied:

1. At $z=2 d_{50}$ with $d_{50}$ the median grain diameter: $C=\max \left(C_{a}, C_{b}\right)$ where $C_{a}$ is a reference concentration obtained by applying instantaneously Engelund and Fredsøe's [1976] formula (quasi-steady approximation) and $C_{b}$ results from particles settling from the upper layers.

2. At $z=z_{h}$, zero flux of sediment reads $\partial C / \partial z=0$. In this paper, we do not discuss the values of the coupling constants $C_{1}, C_{2}$, and $C_{3}$. For the sediment suspension application at the end of the paper, we consider a simplified version of the coupled model after linearization, taking $\Omega=0$. Explanations on the derivation of the coupling constants is given by Lewellen [1977].

\subsection{Modeling of Turbulent Separation Under the Effect of an Adverse Pressure Gradient}

[13] We now discuss the modeling of turbulence separation near flow reversal. Starting from experimental observations in an oscillating tunnel, a stronger turbulent activity shows in Reynolds stress time series (secondary humps) when the pressure gradient is adverse and nearly maximum, i.e., at the end of the decelerating parts of the cycle in the rough fully turbulent regime. Indeed large values of Reynolds stresses are observed for positive velocity under strong positive pressure gradient and for negative velocity under strong negative pressure gradient. In tunnel experiments, the horizontal pressure gradient is equal to the opposite of the outer velocity time derivative and it is then $90^{\circ}$ out of phase with the velocity. The phase for both the oscillatory part of the outer velocity and the corresponding oscillatory pressure gradient are defined on Figure 1. Under the effect of the adverse pressure gradient at the end of the decelerating phases of the cycle, the velocity profiles become more and more S-shaped with a large inflection point and finally a back-flow hereafter called flow separation, is present close to the wall. In the laminar regime, the presence of an inflection point is associated to instability and to transition to turbulence [Foster et al., 1994; Wu, 1992]. In the turbulent regime, separation under the effects of an adverse pressure gradient occurs as well, even though the turbulent boundary layer can resist to separation longer than the laminar boundary layer, at the expenses of an increased wall friction. On the basis of the experimental results, we think that this resistance to separation also depends on the wall roughness, namely a rough wall is more resistant than a smooth wall. Hence, we suggest to model this wall friction enhancement before flow separation under the effect of the adverse pressure gradient for fully developed turbulence and rough walls only, as follows.

[14] First, it is necessary to establish when the enhancement in the wall friction begins. A criterion for the wall shear stress enhancement before turbulent separation under adverse pressure gradient can be found by extending to oscillatory flows the definition of the equilibrium parameter (8) first defined by Clauser [1954] for steady flows:

$$
\beta_{T}(t)=\frac{\delta^{*}(t)}{\tau(t)} \frac{d P}{d x}(t)
$$

where $\delta^{*}(t)=\int_{0}^{\delta}\left(1-\frac{u}{U}\right) d z$ is the instantaneous displacement thickness, $\tau(t)$ is the instantaneous bottom shear stress and $\frac{d P}{d x}(t)$ is the instantaneous pressure gradient. This suggestion, like the use of a Reynolds averaged model, relies on a quasi-steady assumption. Indeed, it is assumed that in such oscillatory flows (with oscillating frequency smaller than turbulence frequencies) it is possible to find a time step for which the outer flow can be considered steady for applying Reynolds averaging and hence define Clauser parameter.

[15] Turbulent separation occurs when $\beta_{T}$ tends to infinity (in practice when $\beta_{T}$ is larger than 20 ), that is, when $\tau=0$. For steady flows, $\beta_{T}$ is positive for adverse pressure gradient and negative for favorable pressure gradient. For oscillatory flows, this classification does no longer hold. Indeed, $\beta_{T}$ is positive under a favorable pressure gradient when the near-wall velocity is negative. Hence, to define the adverse pressure gradient in oscillatory flow, we should compare the pressure gradient action to the near-wall velocity. However, Clauser parameter still tells the relative strength of the pressure gradient compared to the flow. For steady flows, Wilcox ranges the pressure gradient strength from low $\left(\beta_{T}<2\right)$ to moderate $\left(2<\beta_{T}<4\right)$ and strong $\left(\beta_{T}>\right.$ 4). For oscillating flows, we suggest to initiate the wall shear stress enhancement resisting and preceding turbulent separation when the pressure gradient is adverse and 

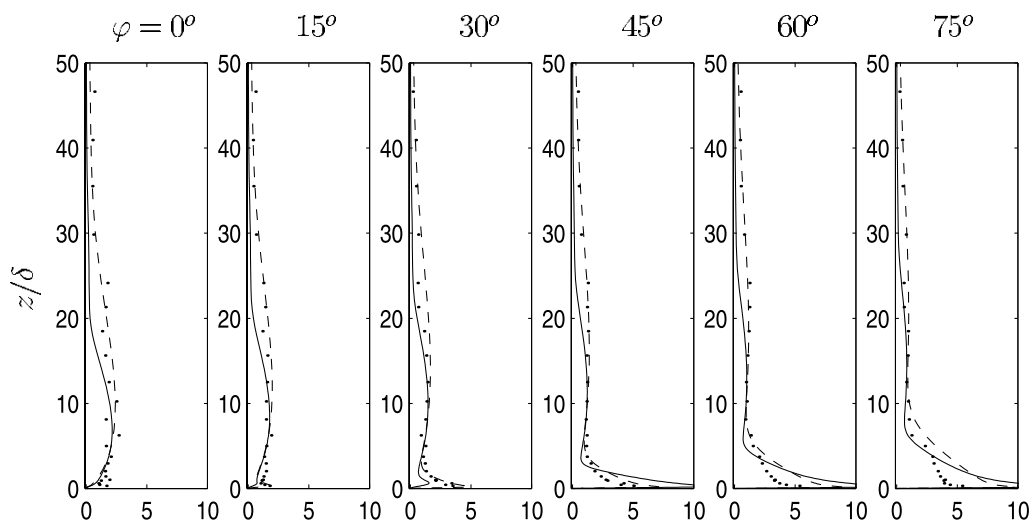

a.

$$
k / U_{0}^{2}
$$
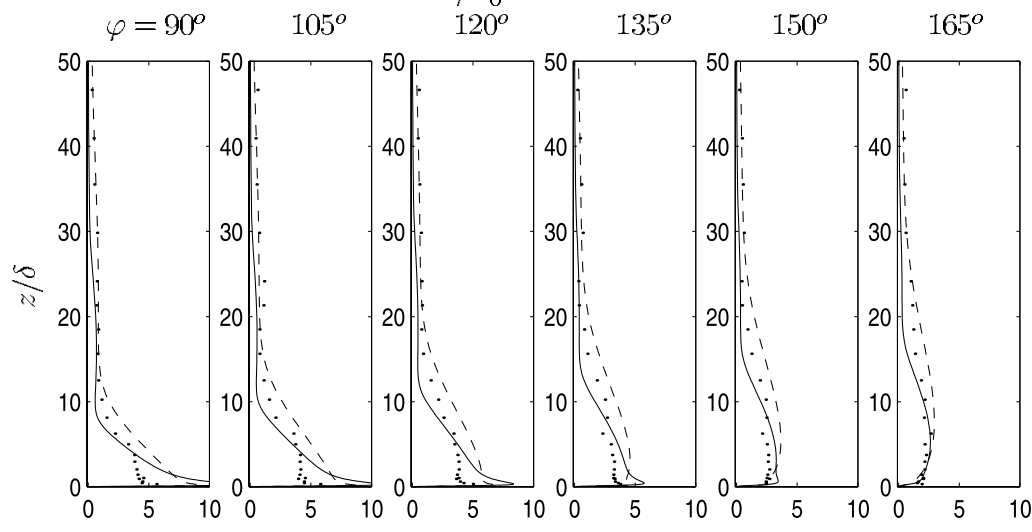

b.

$$
k / U_{0}^{2}
$$

Figure 9. Turbulent kinetic energy vertical profiles at different phases during the accelerating part of the cycle (a) and during the decelerating part of the cycle (b) measured by Jensen et al. [1989, test 8] (...), computed with the original Wilcox transitional $k-\omega$ model (- -) and the new $k$ - $\omega$ model $(-)$ for a sinusoidal outer flow $U=U_{0} \sin (2 \pi t / T)$ with $T=9.72 \mathrm{~s}, U_{0}=1.02 \mathrm{~m} / \mathrm{s}$ over a smooth bottom $\left(R_{\delta}=1789\right.$, $\left.R_{e}=1.6 \times 10^{6}\right)$.

$\left\|\beta_{T}(t)\right\|$ is larger than a threshold value $\beta_{\text {sep }}$ (strong adverse pressure gradient).

[16] Second, we model the wall shear stress enhancement, when these conditions are fulfilled, prescribing a much lower value for the energy dissipation rate at the wall, $\omega_{\text {wall }}$, than the one given by the Wilcox condition cited above [Wilcox, 1998, p. 177]. Indeed, a decrease of $\omega_{\text {wall }}$ leads to an increase of the eddy viscosity and hence to an increase of the Reynolds stress, which is consistent with what is observed in the experiments. In the light of Wilcox's rough wall condition i.e., $\omega_{\text {wall }}=100 u_{f} / k_{N}$, it can be understood that decreasing $\omega_{\text {wall }}$ is a way to take into account, in a 1DV model, the 3-D vortex shedding mentioned by Sleath [1987]. Indeed, we suggest that vortex shedding increases the apparent bottom roughness in relation with the vortex size, and consequently decreases $\omega_{\text {wall }}$. However, and as long as it is not possible to prescribe the dynamics of such an apparent bottom roughness according to the vortex shedding, we suggest a shortcut by imposing a fixed constant low value $\omega_{\text {vortex }}$ for $\omega_{\text {wall }}$. It is worth noticing that the resulting wall shear stress enhancement tends to decrease the value of $\left\|\beta_{T}(t)\right\|$ which would cause a feedback on this condition. This feedback can lead to numerical instabilities in Reynolds stress time series close to the wall if $\omega_{\text {vortex }}$ is much smaller than the usual value for $\omega_{\text {wall }}$. We think it is not relevant to allow for such a feedback as long as the apparent bottom roughness dynamics in relation with the vortex shedding is not included. That is to say that after $\left\|\beta_{T}(t)\right\|$ reaches for the first time $\beta_{\text {sep }}$ during the decelerating parts of the wave cycle, we apply $\omega_{\text {wall }}=$ $\omega_{\text {vortex }}$ until the pressure gradient becomes favorable again. However, a higher time resolution is required in computations to deal with this separation condition under the effect of a strong adverse pressure gradient. Indeed, without the separation condition, reliable computations are obtained with a $1^{\circ}$ phase resolution. In contrast, a $0.1^{\circ}$ phase resolution is required to obtain converged computations with the separation condition.

[17] Concerning spatial resolution, computations are done on a vertical grid whose step size increases exponentially from bottom to top, thus giving a higher resolution near the bed where velocity gradients are important [Huynh-Than and Temperville, 1990]. Computations with near bed step size of $10^{-6}$ and $10^{-7} \mathrm{~m}$ have been compared and give the same results. Thus, for all the computations presented in this paper, a near bed step size of $10^{-6} \mathrm{~m}$ was used, with 173 grid points exponentially distributed over the boundary layer thickness.

[18] We discuss now the model sensitivity to the values chosen for $\beta_{\text {sep }}$ and $\omega_{\text {vortex }}$. On Figures $2 \mathrm{a}$ and $2 \mathrm{~b}$, we plot 

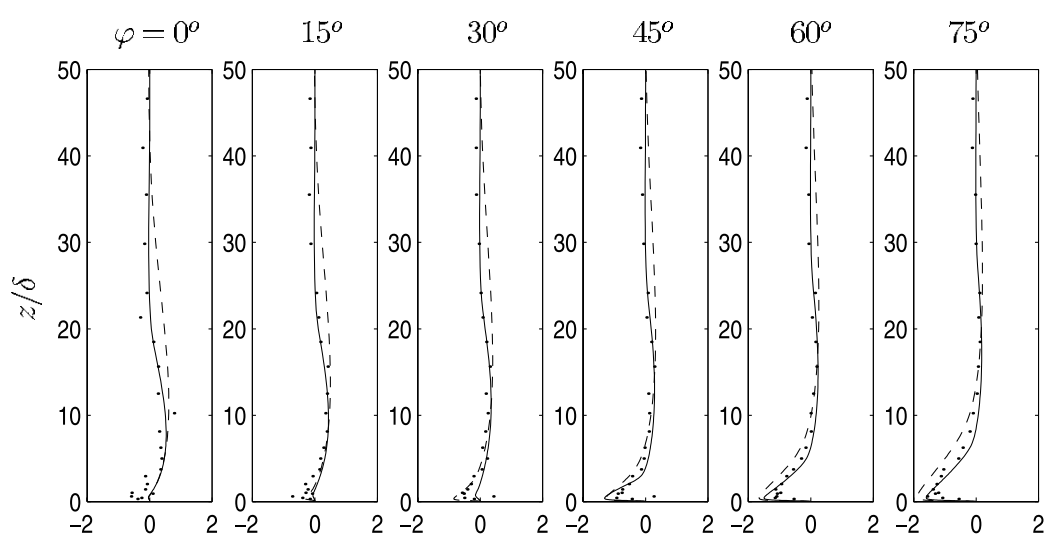

a.

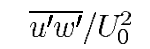

a. $\varphi=90^{\circ}$
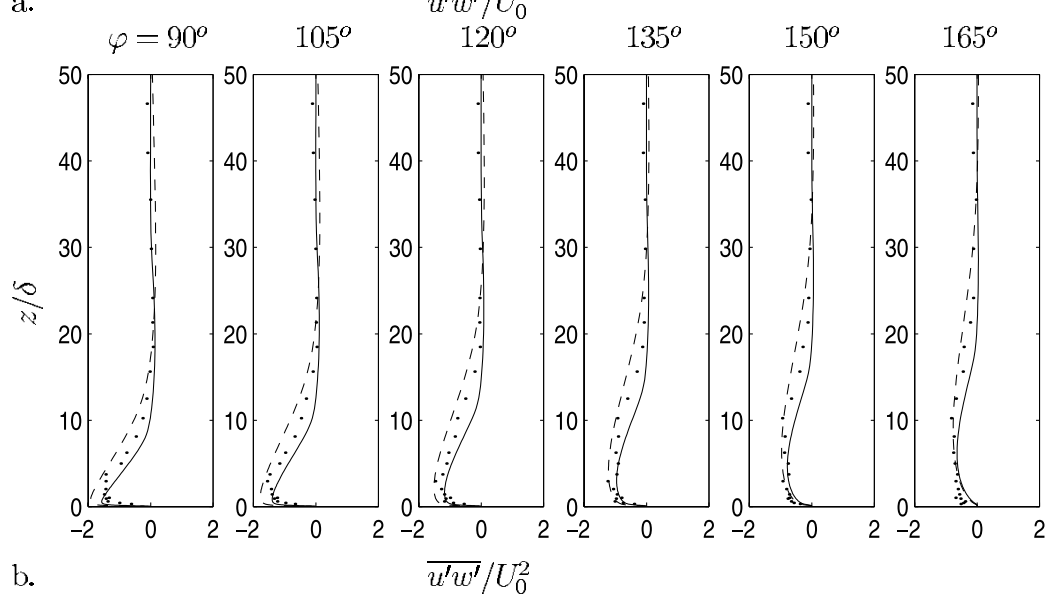

b.

$$
\overline{u^{\prime} w^{\prime}} / U_{0}^{2}
$$

Figure 10. Reynolds stress vertical profiles at different phases during the accelerating part of the cycle (a) and during the decelerating part of the cycle (b) measured by Jensen et al. [1989, test 8] (...), computed with the original Wilcox transitional $k-\omega$ model (- -) and the new $k-\omega$ model ( - ) for a sinusoidal outer flow $U=U_{0} \sin (2 \pi t / T)$ with $T=9.72 \mathrm{~s}, U_{0}=1.02 \mathrm{~m} / \mathrm{s}$ over a smooth bottom $\left(R_{\delta}=\right.$ $\left.1789, R_{e}=1.6 \times 10^{6}\right)$.

computed Reynolds stress time series close to the wall corresponding to the flow condition of Dohmen-Janssen experiment G4 (see section 4.1). For $\beta_{\text {sep }}$ ranging from 4 to 40 , it is clear that the lower $\beta_{\text {sep }}$ is, the earlier shear stress enhancement begins (as can be expected) and the larger shear stress enhancement is. We will see in section 4.1 that a good agreement with tunnel experiments is found for $\beta_{\text {sep }}=20$ which is in line with the usual practical value for turbulent separation. On Figure $2 b$, we show the computations with $\beta_{\text {sep }}=20$ for $\omega_{\text {vortex }}$ ranging from 30 to 3000 (usual values for $\omega_{\text {wall }}$ for this flow condition is $\left.10^{4}\right)$. As expected, the larger $\omega_{\text {vortex }}$ is, the smaller the shear stress enhancement is. We will see in section 4.1 that a good agreement with tunnel experiments is found for $\omega_{\text {vortex }}=300$.

\section{Pure Oscillatory Flow Over a Smooth Bottom}

\subsection{The $\boldsymbol{k}$ - $\boldsymbol{\omega}$ Models Versus DNS}

[19] Vittori and Verzicco [1998], by integrating numerically the Navier Stokes equations (DNS), observed wall shear stress enhancements during the decelerating phases of the oscillatory flow over a smooth bottom for moderate values of the Reynolds number $R_{\delta}=U_{0} \delta / \nu=\sqrt{2 R_{e}}\left(U_{0}\right.$, $T$ are the amplitude and period of the velocity oscillations outside the boundary layer, $\delta=\sqrt{\nu T / \pi}$ is the viscous boundary layer thickness, $v$ is the kinematic viscosity). Vittori and Verzicco [1998] also observed the "disturbedlaminar" and "intermittently turbulent" regimes [Hino et al., 1976] and investigated the transition from these two regimes. The "intermittently turbulent" regime, observed for values of $R_{\delta}$ larger than a critical value which depends on the intensity of the external disturbances but ranges around 600 , shows turbulence activity during the decelerating phases of the cycle, while in the rest of the cycle the flow tends to recover a laminar-like behavior. When turbulent fluctuations start, at the beginning of the decelerating part of the cycle, a sudden strong increase in the wall shear stress is observed which might cause an increase of the sediment pick-up from the bed and an enhancement of the concentration.

[20] Hence, in this subsection, we discuss the ability of the original Wilcox transitional model to predict flow field transition from the laminar to the turbulent regime under oscillatory pressure gradient for smooth wall and moderate Reynolds number conditions. In order to test its performance, a DNS has been performed for the following hydrodynamical conditions: $U_{0}=1.1 \mathrm{~m} / \mathrm{s}, T=4 \mathrm{~s}, R_{\delta}=1241$ and $\delta=1.12810^{-3} \mathrm{~m}$. For details on the DNS, the reader should refer to Vittori and Verzicco [1998]. For these conditions, 
Table 1. Experimental Conditions for Dohmen-Janssen [1999] Clear Water Experiments and Janssen et al. [1997] Sand Experiments

\begin{tabular}{cccccc}
\hline Experiment Name & Grain Size $d_{50}, \mathrm{~mm}$ & Roughness $k_{N}, \mathrm{~m}$ & Current at $z=100 \mathrm{~mm} u_{c}, \mathrm{~m} / \mathrm{s}$ & Wave Velocity $u_{w}, \mathrm{~m} / \mathrm{s}$ & Period $T, \mathrm{~s}$ \\
\hline G4 & fixed bed & $5.25 \times 10^{-4}$ & 0.27 & 1.44 & 7.2 \\
G5 & fixed bed & $5.25 \times 10^{-4}$ & 0.46 & 0.96 & 7.47 \\
H6 & 0.13 & $3.25 \times 10^{-4}$ & 0.24 & 1.47 \\
E2 & 0.21 & $5.25 \times 10^{-4}$ & 0.24 & 7.2 \\
\hline
\end{tabular}

we suggest that flow separation induces the laminar-turbulent transition, the modeling of which is already included in the transitional model.

[21] In the following, the DNS results presented have been phase-averaged in order to remove the stochastic behavior characteristic of a single realization of the process. For details on the averaging procedure, the reader is referred to Vittori and Verzicco [1998] and Costamagna et al. [2002]. In Figure 3b, the wall shear stress computed with the DNS and the Wilcox original transitional model are plotted. Wilcox model is indeed able to predict the wall shear stress enhancement at the laminar-turbulent transition as shown in the DNS computations. In particular, the maximum wall shear stress predicted by the Wilcox original model is close to DNS predictions. However, the initiation of the transition occurs earlier in the Wilcox model than in the DNS. In the transitional $k$ - $\omega$ model, the initiation of this transition is controlled by the two parameters $R_{K}$ and $R_{\omega}$, whereas the amplitude of the bottom shear stress enhancement is linked to the $R_{K} / R_{3}$ ratio. The chosen values of $R_{K}=$ $20, R_{\beta}=27$ and $R_{\omega}=2.95$ lead to much better results (Figure $3 \mathrm{~b}$ ).

[22] Velocity (Figure 3a), Reynolds stress (Figure 4) and turbulent kinetic energy (Figure 5) vertical profiles through the boundary layer at different phases during half oscillation are also plotted. It is noticeable that the original Wilcox model underestimate the vertical decay of all these variables. By changing the value of the diffusion constants in the
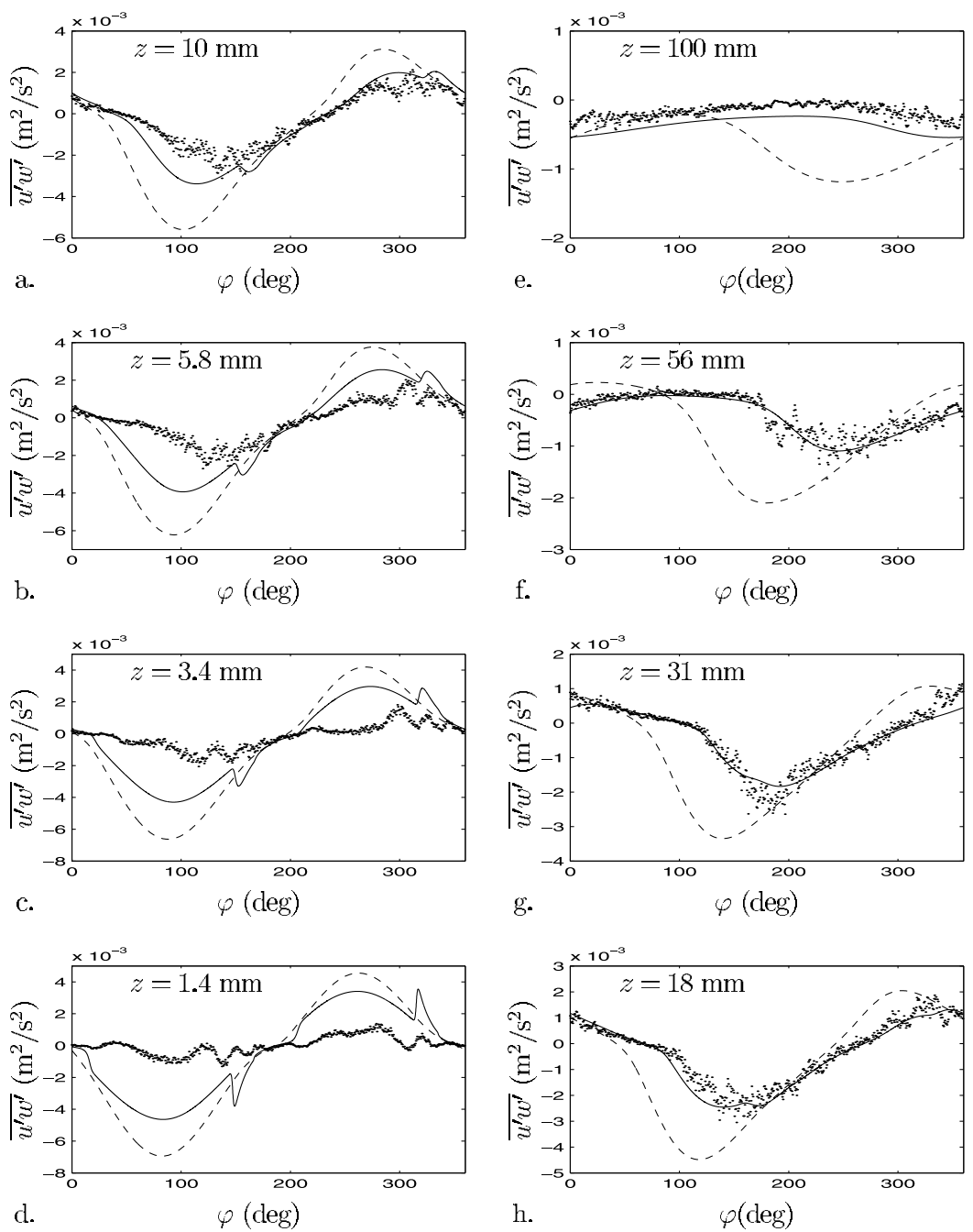

Figure 11. Phase-averaged Reynolds stress time series at different levels from the bed measured by Dohmen-Janssen [1999, exp. G4] (...), obtained using the original Wilcox transitional $k$ - $\omega$ model (- -) and the new $k$ - $\omega$ model $(-)\left(R_{\delta}=2179, R_{e}=2.4 \times 10^{6}, A / k_{N}=3173\right)$. 

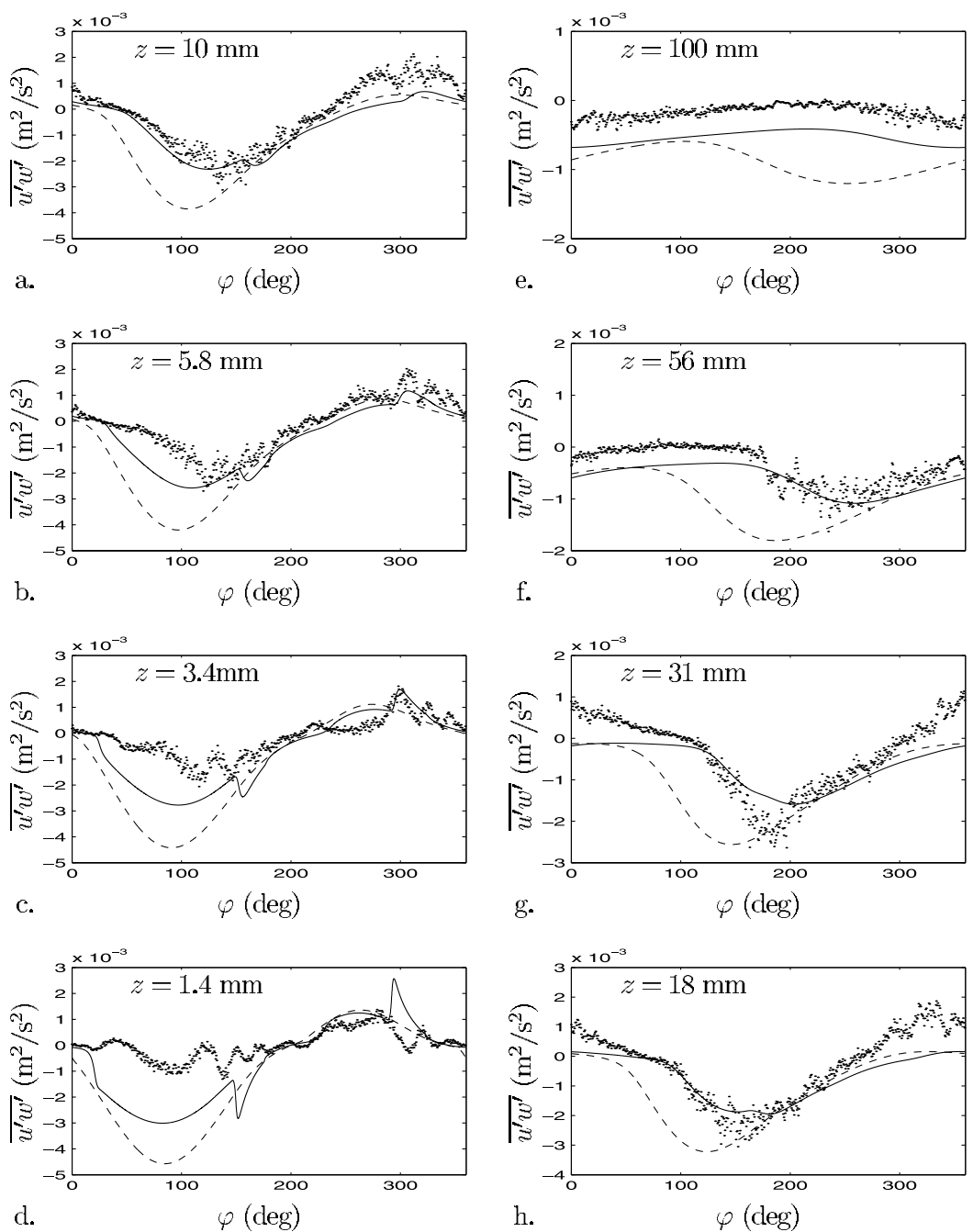

Figure 12. Phase-averaged Reynolds stress time series at different levels from the bed measured by Dohmen-Janssen [1999, exp. G5] (...), obtained using the original Wilcox transitional $k$ - $\omega$ model (- -) and the new $k$ - $\omega$ model $(-)\left(R_{\delta}=1453, R_{e}=1.06 \times 10^{6}, A / k_{N}=2115\right)$.

$k$ and $\omega$-equations (taking $\sigma^{*}=0.8$ and $\sigma=0.375$ ), we can improve the $k-\omega$ model results for the vertical decay of the velocity, the Reynolds stress and the turbulent kinetic energy compared to DNS results. The agreement between the so-called new $k$ - $\omega$ model and the DNS is indeed remarkable except close to the wall $(z \leq 5 \delta)$ for the turbulent kinetic energy. Indeed, the original Wilcox model gives better estimation of the near-wall turbulent kinetic energy. Meanwhile, these near wall discrepancies, especially in turbulent kinetic energy predictions, have reduced effects on the wall shear stress prediction (Figure $3 b$ ).

\subsection{The $k-\omega$ Models Versus}

\section{Jensen et al. [1989] Experiments}

[23] In this section, we compare predictions of the two transitional models with the experimental measurements of Jensen et al. [1989], which were made within the boundary layer of a pure oscillating flow over a smooth bottom. Details concerning these experiments are given by Jensen et al.

[24] Jensen et al. carried out experiments using a smooth wall for various Reynolds number ranging from the laminar regime $\left(R_{e}=3.3 \times 10^{4}\right)$ up to the fully turbulent regime $\left(R_{e}=6.0 \times 10^{6}\right)$, and in particular, they measured bottom shear stress. On Figure 6, the nondimensional bottom shear stress time series (bottom shear stress time series divided by the maximum bottom shear stress) computed using the original Wilcox model and the new one are plotted. For the sake of clearness, we do not show Jensen et al.'s measurements on this graph. To compare the theoretical predictions with the experimental data, this figure should be compared to Figure 9 of Jensen et al. It is then clear that in the original Wilcox model, the laminar-turbulent transition develops much quicker for $R_{e}$ larger than $3.3 \times 10^{4}$, whereas the new model with modified value for $R_{K}$ and $R_{\beta}$ gives results closer to the measurements. This is confirmed by Figure 7 where the computed dimensional bottom shear stresses are plotted along with the measured values. However, it can be noticed that in the measurements for $R_{e}$ larger than $6.0 \times 10^{6}$, turbulence seems to have pervaded the whole cycle whereas in the new model results the boundary layer still recovers its laminar behavior at the very beginning of the accelerating phases of the cycle, even though for these 

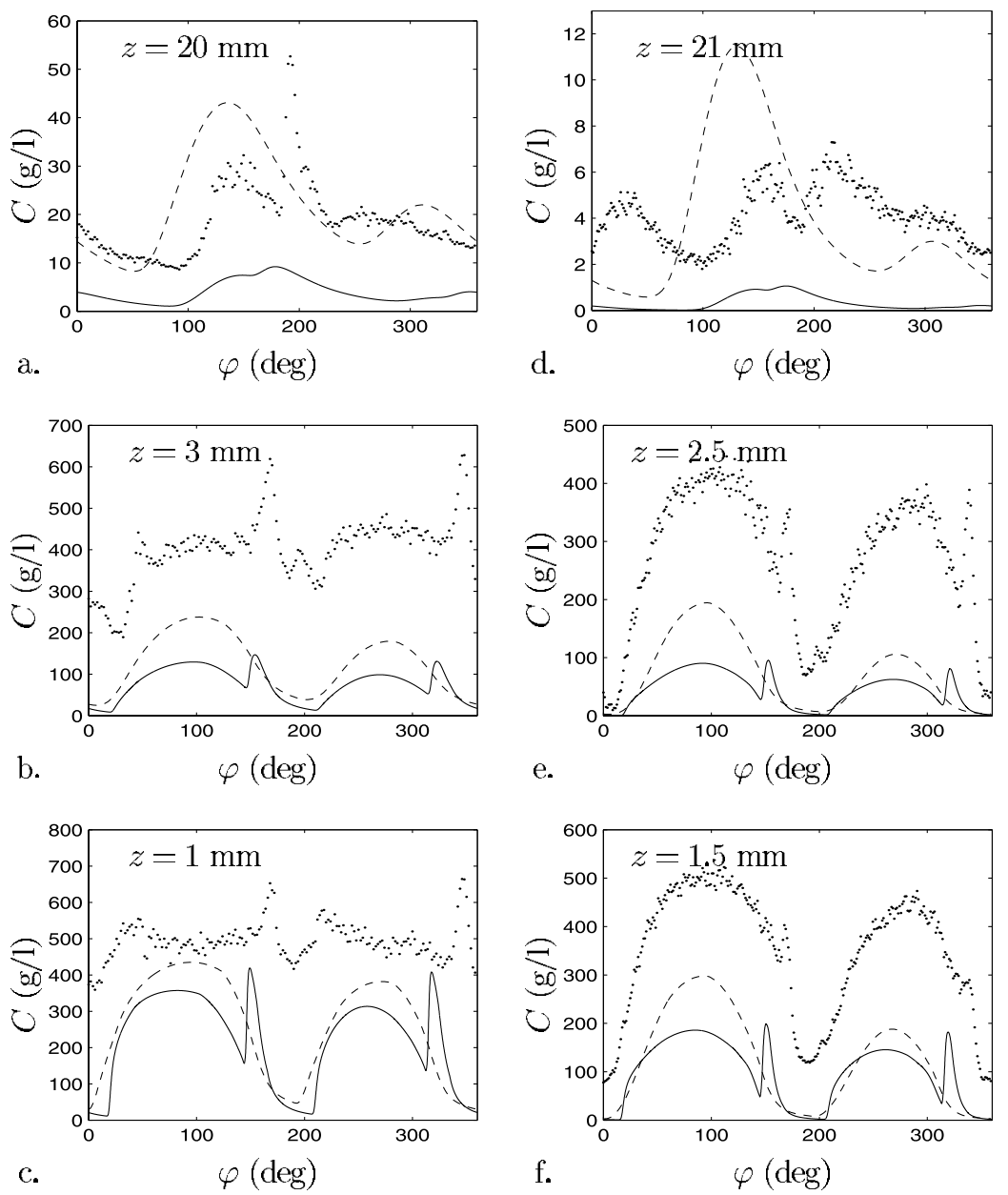

Figure 13. Phase-averaged concentration time series measured by Janssen et al. [1997, exp. E2 and H6] (...) and computed with the original Wilcox transitional $k$ - $\omega$ model (- -) and the new $k$ - $\omega$ model (-) (in both models, turbulence damping by stratification is included) for $d_{50}=0.13 \mathrm{~mm}$ at $z=1 \mathrm{~mm}$ (c), $z=$ $3 \mathrm{~mm}$ (b) and at $z=200 \mathrm{~mm}$ (a) from the bottom and for $d_{50}=0.21 \mathrm{~mm} z=1.5 \mathrm{~mm}$ (f), $z=2.5 \mathrm{~mm}$ (e) and at $z=210 \mathrm{~mm}$ (d) from the bottom. Hydrodynamical conditions are given in Table 1 .

low value of the outer flow velocity, the difference between fully turbulent and laminar value of the bottom shear stress are small.

[25] Then we compare measured and modeled characteristics of the boundary layer for a specific experiment, in particular test 8 from Jensen et al. $\left(R_{e}=1.6 \times 10^{6}\right.$, $\left.R_{\delta}=1789\right)$ is considered. This test was also chosen by Thais et al. [1999] to test the Chien low-Reynoldsnumber $k-\varepsilon$ model. Hence, this comparison enables a cross discussion of the results of all the models. The comparison plots are presented with the outer flow scaling. Vertical profiles through the boundary layer at different phases during half cycle are plotted for the velocity (Figure 8), turbulent kinetic energy (Figure 9) and Reynolds stress (Figure 10). On these plots, we clearly see that the original Wilcox model does not correctly reproduce the vertical dependency on these three quantities. The better achievements of the new transitional $k$ - $\omega$ model and in particular the improved vertical decay description of the velocity, turbulent kinetic energy and Reynolds stress are obtained by changing the diffusion constants.
[26] However, some discrepancy is present when a detailed comparison of the model results is made with the experimental data. Part of this discrepancy might be due to experimental errors. In particular, turbulent quantities measured using the laser Doppler anemometer (LDA) may be underestimated near the wall. Indeed, according to the Prandtl mixing length theory the turbulent length scale is equal to $\kappa z$, with $\kappa$ the Von Kármán constant $(\kappa=0.4)$. Hence, part of the turbulence may be averaged out over the sampling volume of the LDA when turbulent length scale is smaller than the size of the sampling volume. In Jensen et al. experiments, the sampling volume sizes are $0.15 \mathrm{~mm} \times 0.15 \mathrm{~mm} \times 2.5 \mathrm{~mm}$. This means that up to $z=3.5 \delta=6.25 \mathrm{~mm}$, turbulent quantities might be underestimated. This might explain why the measured turbulent kinetic energy is smaller than even the Wilcox model predictions close to the wall at phases around flow reversal $\left(\varphi=2 \pi t / T=0,15,30,150\right.$ and $\left.165^{\circ}\right)$. Another error source might be due to the prescription in the models of an exact sinusoidal outer flow whereas in the measurements, the outer flow is not perfectly sinusoidal. Finally, it 

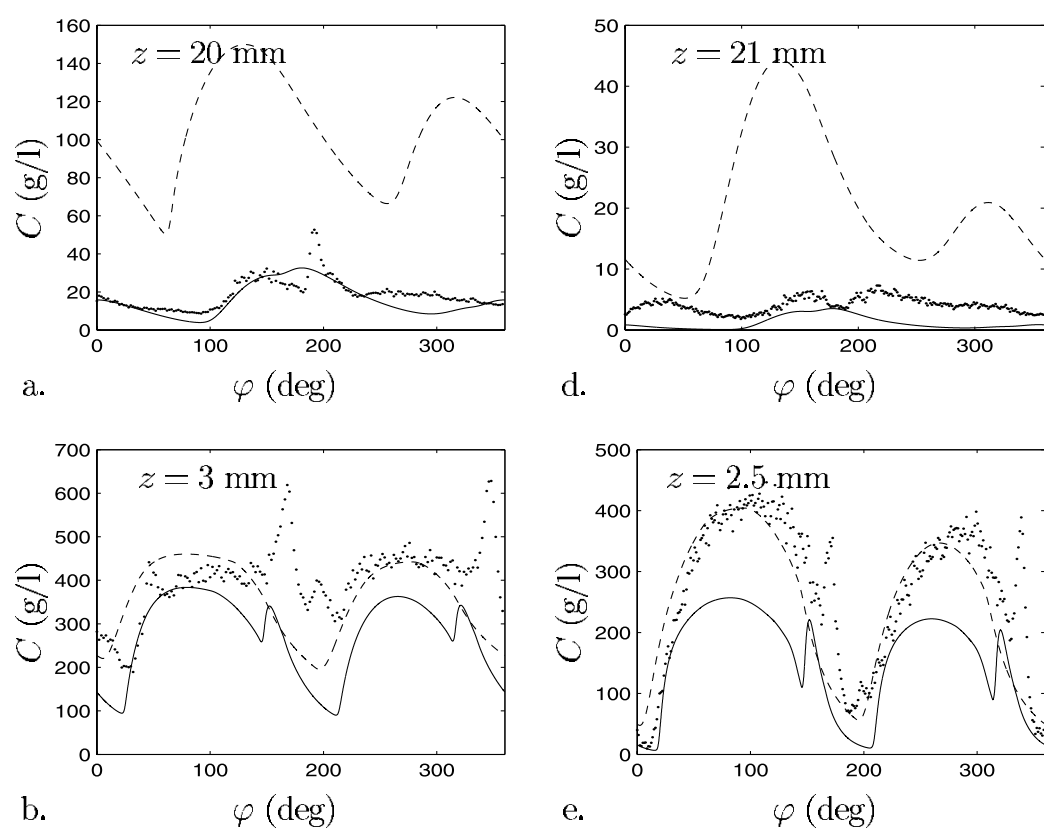

d.

$$
\varphi(\mathrm{deg})
$$
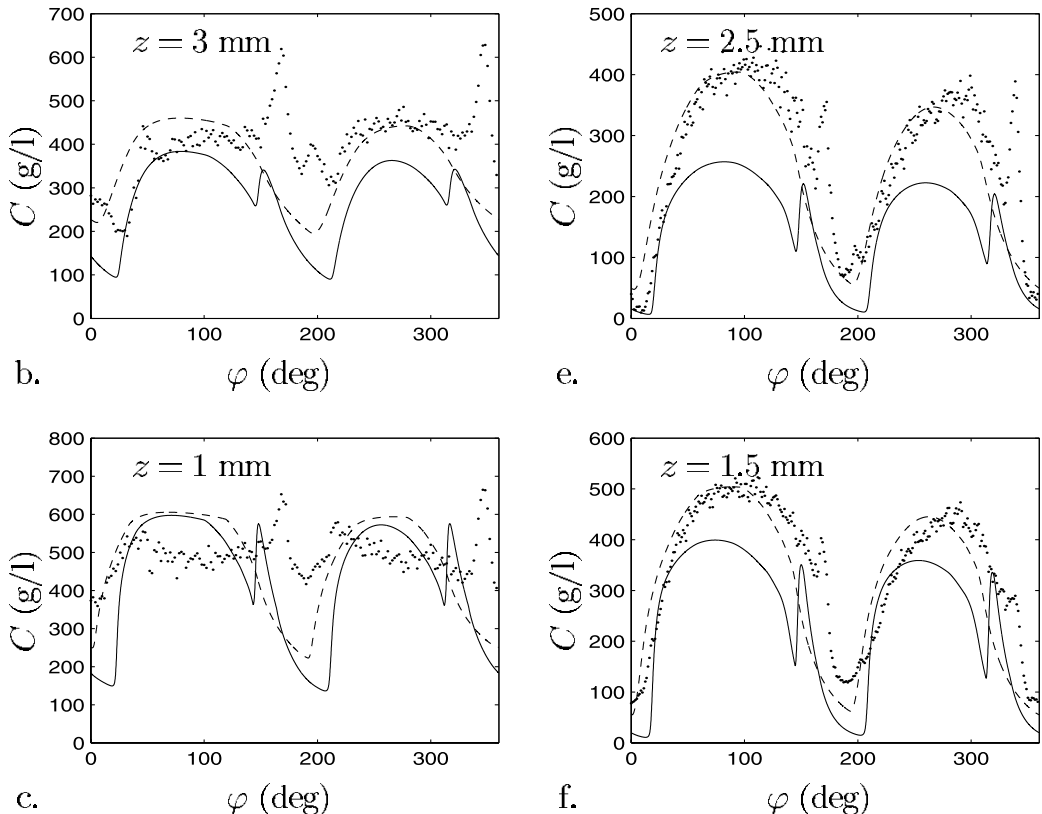

Figure 14. Same legend as Figure 13, but in both models, enhanced hindering for high concentration in the sheet flow layer has been introduced.

should be recognized that the Chien low-Reynolds-number model used by Thais et al. [1999] performs also very well in comparison with this data set.

\section{Oscillatory Flow Plus Current Over a Rough Bottom: The $k-\omega$ Model Versus Tunnel Experiments}

\subsection{Dohmen-Janssen [1999] Clear Water Experiments}

[27] The experiments were carried out in the large oscillating water tunnel (LOWT) of WL||Delft Hydraulics. For a detailed description of the LOWT, the reader can refer to Ribberink and Al Salem [1994]. This facility enables a full-scale simulation of the horizontal orbital motion near the bed associated to waves. The LOWT is a U-shaped tube, with a piston in one of the cylindrical risers to generate a horizontal oscillatory flow in the test section. Due to this configuration, vertical orbital velocities are not generated in the tunnel. A recirculation system allows the generation of a net current in addition to the oscillatory flow. The test section is $0.3 \mathrm{~m} \times 0.8 \mathrm{~m}$ by $12 \mathrm{~m}$ long. For clear water experiments, a fixed rough bottom was used, made of glued sand of diameter $d_{50}=$ $0.21 \mathrm{~mm}$ on a wooden bottom. Time-dependent flow velocities, including turbulent components, were measured with a two-component LDA in forward-scatter mode. The LDA has a relatively small sampling volume, with a height and width (in flow direction) of only $0.215 \mathrm{~mm}$. The width in cross direction is larger $(6.47 \mathrm{~mm})$. The experimental conditions of the clear water experiments we first consider in this section are named G4 and G5 and given in Table 1.

[28] Figures 11 and 12 show the Reynolds stress time series measured by Dohmen-Janssen [1999] at various levels above the rough bed (experiments G4 and G5), along with the computations obtained by Wilcox transitional $k$ - $\omega$ model and our new $k$ - $\omega$ model taking $\beta_{\text {sep }}=$ 20 and $\omega_{\text {wall }}=300$. We clearly observe on Figures 11a, $11 \mathrm{~b}$, and $11 \mathrm{c}$, secondary humps at the end of the decelerating phase in the measured Reynolds stress time series. These humps are also present in our new model computations. Yet, the computed values of Reynolds stress are larger than those measured up to $z=10$ $\mathrm{mm}$. In fact, the experimental values might be underestimated due to the size of the sampling volume of the LDA as explained in section 3.2. This means that up to $z=16 \mathrm{~mm}$, part of the turbulence may be averaged out, resulting in smaller value of $\overline{u^{\prime} w^{\prime}}$. In addition, we think the values we suggest for $\omega_{\text {wall }}$ and $\beta_{\text {sep }}$ to model secondary humps at the end of the decelerating phase 
will give physical and realistic results for usual field conditions since experiments G4 and G5 correspond to drastic field conditions. Above $z=10 \mathrm{~mm}$, the agreement between the measured and the new model's results is noticeable on both the amplitude and the phase predictions. In contrast, the Wilcox model mainly fails on the phase prediction. The better performance of the new model in the phase prediction is related to the better description of the vertical decay of Reynolds stress, velocity and turbulent kinetic energy as already noticed when the model predictions were compared with the DNS. This finding is a further confirmation of the better choice for the diffusion constants $\sigma$ and $\sigma^{*}$ in our new model.

\subsection{Janssen et al. [1997] Experiments With Sediments}

[29] Hereafter, we present an application of the new $k-\omega$ model described in section 2 to predict sediment suspension. For this purpose, we compare the results obtained using the original Wilcox transitional model and the new $k-\omega$ model. In both models, turbulence damping by the stratification induced by suspended sediment is included as described in section 2. The concentration equation is thus solved simultaneously with the hydrodynamics using Engelund and Fredsøe's [1976] reference concentration condition at the bottom. We apply the two models for the test conditions H6 and E2 of Janssen et al. [1997] (see Table 1). In Figure 13 the measured and modeled concentration time series taken at different levels above the bed are shown. Concentration measurements shows sharp increases before flow reversal near the bottom (Figures 13b, 13c, 13e, and 13f). Doubts can be cast on these sudden concentration peaks that could be linked to the measuring technique. Indeed, a sediment deposition between the electrodes of the conductivity probe $(\mathrm{CCM})$ at low velocity could cause such a signal. Nevertheless, concentration peaks are also observed in time series measured using optical conductivity probes (Figures 13a and 13d) further from the bottom. Hence, we feel that the peaks in the measured concentration time series are the effective signature of suspension ejection events, although a possible bias in $\mathrm{CCM}$ probes measurements should be investigated. The separation condition introduced in the new transitional $k-\omega$ model enables to reproduce the secondary peaks observed in measured concentration time series before flow reversal. Moreover, by changing the diffusion constants, we have improved the description of the phase concentration in the upper part of the boundary layer. However, a significant discrepancy still remains between the predicted and the measured values. Indeed, the concentration is underestimated throughout the entire boundary layer. It should be stressed that the overall concentration underestimation in the new model contrast with the traditional model predictions. Indeed, the traditional model, despite its underestimation of the concentration in the lower levels, agrees better with the data at $z=20 \mathrm{~mm}$. In our opinion, this better agreement of the traditional model is coincidental. Indeed, discrepancies between the lower and upper levels is in line with the fact that the Reynolds stress vertical decay is not correctly predicted by the traditional model (as shown in comparison with the DNS in Figure 4). In contrast, in the new model, the concentration is underestimated at all levels (see Figure 13). The model predictions can be improved at all levels by taking into account the intergranular forces in the "sheet flow" layer (highly concentrated bottom layer). Indeed, following Guizien et al.'s [2001] suggestion of enhancing the hindering in the sheet flow layer i.e., taking a lower settling velocity for volume concentration larger than 0.01 , the agreement of the new model with the data is improved at all levels as shown in Figure 14. In contrast, in the traditional model, although the agreement is improved at the lower levels, it turns worst in the upper levels (see Figures 14a and 14b). This is consistent with the improvement in the new model predictions of the turbulence vertical decay as already mentioned in section 3.1. However, discrepancies still remains between predicted and measured values. We suggest this could be improved by looking into more details in the modeling of the intergranular forces in the "sheet flow" layer and also in the sediment feedback on turbulence, but this is beyond the scope of this paper.

\section{Conclusions}

[30] A new transitional $k$ - $\omega$ model has been devised introducing a turbulent separation condition under adverse pressure gradient and modifying the diffusion and transition constants of the Wilcox [1992] original $k-\omega$ transitional model. The constants modification is suggested by a comparison with a D.N.S computation of a pure oscillatory flow over a smooth bottom in the laminarturbulent transitional regime $\left(R_{e}=7.7 \times 10^{5}\right)$. The new model is then validated by comparing its predictions with experimental data by Jensen et al. [1989] for Reynolds number $R_{e}$ ranging from $3.3 \times 10^{4}$ to $6 \times 10^{6}$. In this regime, flow separation induces the transition from the disturbed-laminar to the intermittently turbulent regimes, which is modeled by the dependence of the constants of the high-Reynolds Wilcox [1988] model upon a local Reynolds number. We are thus able to reproduce the wall shear stress sharp increase, which takes place at transition in good agreement with Jensen et al. data. The change of the diffusion constants improves also the description of the vertical distribution of both velocity and Reynolds stress compared to the original transitional Wilcox [1992] model. The new model gives fairly good results, except concerning the value of the turbulent kinetic energy close to the wall around $\varphi=90^{\circ}$, and is low-time consuming.

[31] The change of the diffusion and transition constants proposed in this new model also enables to improve the description of Reynolds stress vertical distribution in the fully rough turbulent regime. Indeed, as shown by a comparison with experimental data in clear water, the model reproduces accurately the vertical phase lagging of Reynolds stress. In addition, for these experimental conditions, the turbulent separation condition acts and enables to reproduce the secondary humps in the measured Reynolds stress time series. This feature has never been reproduced in standard R.A.N.S. models. Finally, we use the validated new $k-\omega$ model to predict sediment transport and compare the results with experimental data. By solving the concen- 
tration convective-diffusive equation with turbulence damping, this new model reproduces secondary peaks in concentration time series before flow reversal in accordance with the measurements of Janssen et al. [1997]. However, it should be outlined that the concentration is still underestimated in the sheet flow layer. This finding stimulate us to go on working on this important issue for morphodynamics predictions.

[32] Acknowledgments. This work was funded by the EC through a MAST-III project SEDMOC (contract MAS3-CT97-0115). The first author would like to thank the Instituto do Mar (Universidade de CoimbraPortugal) for awarding a postdoctoral grant.

\section{References}

Chien, K. Y., Predictions of channel and boundary layer flows with a lowReynolds-number turbulence model, AIAA J., 20, 33-38, 1982.

Clauser, F. H., Turbulent boundary layers under adverse pressure gradients, J. Aeronaut. Sci., 21, 91-108, 1954.

Costamagna, P., G. Vittori, and P. Blondeaux, Coherent structures in oscillatory boundary layers, J. Fluid Mech., 474, 1-33, 2002.

Davies, A. G., J. S. Ribberink, A. Temperville, and J. A. Zyserman, Comparison between sediment transport models and observations made in wave and current flows above plane beds, Coastal Eng., 31, 163-198, 1997.

Dohmen-Janssen, M., Grain size influence on sediment transport in oscillatory sheet flow, Ph.D. thesis, Delft Tech. Univ., Delft, Netherlands, 1999.

Engelund, F., and J. Fredsøe, A sediment transport model for straight alluvial channel, Nord. Hydrol., 7, 293-306, 1976.

Foster, D. L., R. A. Holman, and R. A. Beach, Sediment suspension events and shear instabilities in the bottom boundary layer, in Proc. Coastal Dyn. '94, pp. 712-726, ASCE, Barcelona, Spain, 1994.

Fredsøe, J., Turbulent boundary layer in wave-current interaction, $\mathrm{J}$. $\mathrm{Hy}$ drol. Eng., 110(HY8), 1103-1120, 1984

Guizien, K., P. Silva, and F. J. Seabra-Santos, Mathematical modeling of sand transport by combined waves and current in the sheet flow regime using R. A. N. S. turbulence models: Hindering and flow reversal ejections, in SEDMOC Book, pp. 1-8, Aqua Publ., Amsterdam, Netherlands, 2001.

Hino, M., M. Sawamoto, and S. Takasu, Experiments on transition to turbulence in an oscillatory pipe flow, J. Fluid Mech., 75, 193-207, 1976.

Huynh-Than, S., and A. Temperville, A numerical model of the rough turbulent boundary layer in combined wave and current interaction, in Proceedings of the 22nd Coastal Engineering Conference, pp. 853-866, Am. Soc. Civ. Eng., Reston, Va., 1990.

Huynh-Than, S., T. Tran Thu, and A. Temperville, A numerical model for suspended sediment in combined currents and waves, in Sediment Transport Mechanisms in Coastal Environments and Rivers, EUROMECH 310, edited by M. Belorgey, R. D. Rajaona, and J. F. A. Sleath, pp. 122-130, World Sci., Singapore, 1994.

Janssen, C. M., W. N. Hassan, R. J. van der Wal, and J. S. Ribberink, Grainsize influence on sand transport mechanisms, in Proc. Coastal Dyn. '97, Plymouth, pp. 58-68, 1997.
Jensen, B. L., B. M. Sumer, and J. Fredsøe, Turbulent oscillatory boundary layers at high Reynolds numbers, J. Fluid Mech., 206, 265-297, 1989. Jones, W. P., and B. E. Launder, The prediction of laminarization with a two-equation model of turbulence, Int. J. Heat Mass Transfer, 15, 301314,1972

Katapodi, I., J. S. Ribberink, P. Ruol, R. Koelewijn, C. Lodhal, S. Longo, A. Crosato, and H. Wallace, Intra-wave sediment transport in an oscillatory flow superimposed on a mean current, Rep. H-1684, Part III, Eur. Comm. DGXII, Delft, Netherlands, 1994.

Lewellen, W. S., Use of invariant modeling, in Handbook of Turbulence, vol. 1, Plenum, New York, 1977.

Li, Z., and A. G. Davies, Towards predicting sediment transport in combined wave-current flow, J. Waterw. Port Coastal Ocean Eng., 122(4), 157-164, 1996.

Patankar, S. V., Numerical Heat Transfer and Fluid Flows, McGraw-Hill, New York, 1980

Ribberink, J. S., and A. A. Al Salem, Sediment transport in oscillatory boundary layers in cases of rippled beds and sheet flow, J. Geophys. Res., 99(C6), 12,707-12,725, 1994.

Ribberink, J. S., and A. A. Al Salem, Sheet flow and suspension of sand in oscillatory boundary layers, Coastal Eng., 25, 205-225, 1995.

Rodi, W., Turbulence models and their application in hydraulics, in IAHR Monogr., Int. Assoc. for Hydraul. Res., Delft, Netherlands, 1980

Saffman, P. G., A model for in-homogeneous turbulent flow, Proc. R. Soc. London, Ser. A, 317, 417-433, 1970.

Sana, A., and H. Tanaka, Review of $k-\epsilon$ models to analyze oscillatory boundary layers, J. Hydrol. Eng., 126(9), 701-710, 2000.

Savioli, J., and P. Justesen, Sediment in oscillatory flows over a plane bed, J. Hydraul. Res., 35(2), 177-190, 1996.

Sleath, J. F. A., Turbulent oscillatory flow over rough bed, J. Fluid Mech., 182, 369-409, 1987

Spalart, P. R., and B. S. Baldwin, Direct simulation of a turbulent oscillating boundary layer, Turbul. Shear Flows, 6, 417-440, 1989.

Thais, L., G. Chapalain, and H. Smaoui, Reynolds number variation in oscillatory boundary layers, part I, Purely oscillatory motion, Coastal Eng., 36, 111-146, 1999.

Vittori, G., and R. Verzicco, Direct simulation of transition in an oscillatory boundary layer, J. Fluid Mech., 371, 207-232, 1998.

Wilcox, D. C., Reassessment of the scale-determining equation for advanced turbulence models, AIAA J., 26(11), 1299-1310, 1988.

Wilcox, D. C., The remarkable ability of turbulence model equations to describe transition, presented at Fifth Symposium on Numerical and Physical aspects of Aerodynamical Flows, 13 - 15 January, Calif. State Univ., Long Beach, Calif., 1992.

Wilcox, D. C., Turbulence modeling for CFD, 2nd ed., DCW Ind., Inc, La Canada, Calif., 1998.

$\mathrm{Wu}, \mathrm{X}$., The nonlinear evolution of high-frequency resonant triad waves in an oscillatory Stokes layer at high Reynolds number, J. Fluid Mech., 245, 553-597, 1992.

M. Dohmen-Janssen, Department of Civil Engineering, University of Twente, PO Box 217, 7500 AE Enschede, Netherlands.

K. Guizien, Laboratoire d'Océanographie Biologique de Banyuls, BP 44, 66651 Banyuls sur Mer Cedex, France. (guizien@obs-banyuls.fr)

G. Vittori, Dipartimento di Ingegneria Ambientale, Università di Genova, Via Montallegro 1, 16145 Genova, Italy. 